\title{
The Lunar Crater Observation and Sensing Satellite (LCROSS) Payload Development and Performance in Flight
}

\author{
Kimberly Ennico • Mark Shirley • Anthony Colaprete • \\ Leonid Osetinsky
}

Received: 8 October 2010 / Accepted: 25 January 2011 / Published online: 19 February 2011

(C) US Government 2011

\begin{abstract}
The primary objective of the Lunar Crater Observation and Sensing Satellite (LCROSS) was to confirm the presence or absence of water ice in a permanently shadowed region (PSR) at a lunar pole. LCROSS was classified as a NASA Class D mission. Its payload, the subject of this article, was designed, built, tested and operated to support a condensed schedule, risk tolerant mission approach, a new paradigm for NASA science missions. All nine science instruments, most of them ruggedized commercial-off-the-shelf (COTS), successfully collected data during all in-flight calibration campaigns, and most importantly, during the final descent to the lunar surface on October 9, 2009, after 112 days in space. LCROSS demonstrated that COTS instruments and designs with simple interfaces, can provide high-quality science at low-cost and in short development time frames. Building upfront into the payload design, flexibility, redundancy where possible even with the science measurement approach, and large margins, played important roles for this new type of payload. The environmental and calibration approach adopted by the LCROSS team, compared to existing standard programs, is discussed. The description, capabilities, calibration and inflight performance of each instrument are summarized. Finally, this paper goes into depth about specific areas where the instruments worked differently than expected and how the flexibility of the payload team, the knowledge of instrument priority and science trades, and proactive margin maintenance, led to a successful science measurement by the LCROSS payload's instrument complement.
\end{abstract}

Keywords Moon · Mission · Spectroscopy $\cdot$ Impactor $\cdot$ LCROSS $\cdot$ LRO · Class D

\section{Introduction and Mission Overview}

The Lunar Crater Observation and Sensing Satellite (LCROSS) mission's objective was to sample and analyze regolith from a permanently shadowed region (PSR) on the Moon, providing the first "in-situ" study of a PSR. LCROSS had four science goals: (1) Confirm

K. Ennico $(\bowtie) \cdot$ M. Shirley $\cdot$ A. Colaprete $\cdot$ L. Osetinsky

NASA Ames Research Center, Mail Stop N245-6, Moffett Field, CA 94035-1000, USA

e-mail: Kimberly.Ennico@nasa.gov 
the presence or absence of water ice in a PSR on the Moon; (2) Identify the form/state of hydrogen observed at the lunar poles; (3) Quantify, if present, the amount of water in the lunar regolith with respect to hydrogen concentrations; and (4) Characterize the lunar regolith within a permanently shadowed crater on the Moon.

LCROSS launched with the Lunar Reconnaissance Orbiter (LRO) (Vondrak et al. 2010) from Cape Canaveral, Kennedy Space Center, June 18, 2009, 21:32:00 UTC (Fig. 1a). After LRO separation, LCROSS remained connected to the Atlas V upper-stage Centaur rocket. LCROSS completed 112 days in a Lunar Gravity Assist Lunar Return Orbit, during which on-orbit science payload calibrations, shepherding spacecraft (S-S/C) health checks, and trajectory maneuver corrections were performed. The separation maneuver of the S-S/C from the Centaur occurred on October 9, 2009, 01:50:00 UTC.

The mission had two kinetic impact events: (1) the spent $\sim 2300 \mathrm{~kg}$ Atlas V upper-stage Centaur rocket and (2) the $\sim 625 \mathrm{~kg}$ (dry mass plus computed remaining fuel on board at time of impact) LCROSS S-S/C, following the Centaur four minutes later, both traveling at $\sim 2.5 \mathrm{~km} / \mathrm{s}$. The Centaur impacted the moon with more than 200 times the energy of the Lunar Prospector impact (Barker et al. 1999). Impacts within the lunar South Pole Cabeus crater occurred on October 9, 2009 at 11:31:19.506 UTC and 11:35:36.116 UTC, for the Centaur and S-S/C, respectively (Marshall et al. 2011). The mission was managed and operated by NASA Ames Research Center (ARC) with industry partner Northrop Grumman, Space Technology (NGST) and Technical Services (NGTS). Data from the LCROSS payload revealed that the area impacted by the LCROSS Centaur contains significant water and other volatiles (Colaprete et al. 2010).

\section{Payload Measurement Goals and Science Requirements Trace}

The LCROSS mission measurement goals, in order to meet the mission's science goals, were to: (1) Measure the total concentration of water in the sunlit ejecta cloud; (2) Detect any possible hydrogen bearing compounds including water, hydrated minerals, and organics, including hydrocarbons, in the sunlit ejecta cloud; (3) Constrain the sunlit ejecta cloud particle physical properties, including composition and particle size; and (4) Constrain the mechanical properties of the impact site, including minimum regolith depth and strength. Because the LCROSS mission was a mission of opportunity as a secondary payload to the LRO mission, the mission was extremely cost and scheduled constrained. As such, these primary measurement goals as addressed by the payload on the S/S-C were designated as part of the full mission success criteria and not a part of the minimum success criteria (at the Program level). This allowed for a much more streamlined mission assurance approach to the S/S-C payload that would not have otherwise been possible. For example, commercial instruments and parts were allowed with minimal screening (rather than the use of flight qualified parts which would have had much greater cost and longer procurement schedule). To militate against the lessened mission assurance applied to the payload, the LCROSS on-board science payload used multiple measurement techniques with measurement goal overlap. Table 1 summarizes the project Level 3 science requirements that drove the payload design. Table 2 shows the trace between the science payload instruments and the four LCROSS science measurement goals mentioned above and these requirements. Addressing each measurement goal with overlapping techniques and instruments achieved a level of robustness against misinterpretation and minimized mission susceptibility to false positive/negative results and mitigated against possible instrument failure. As seen in Table 2, 


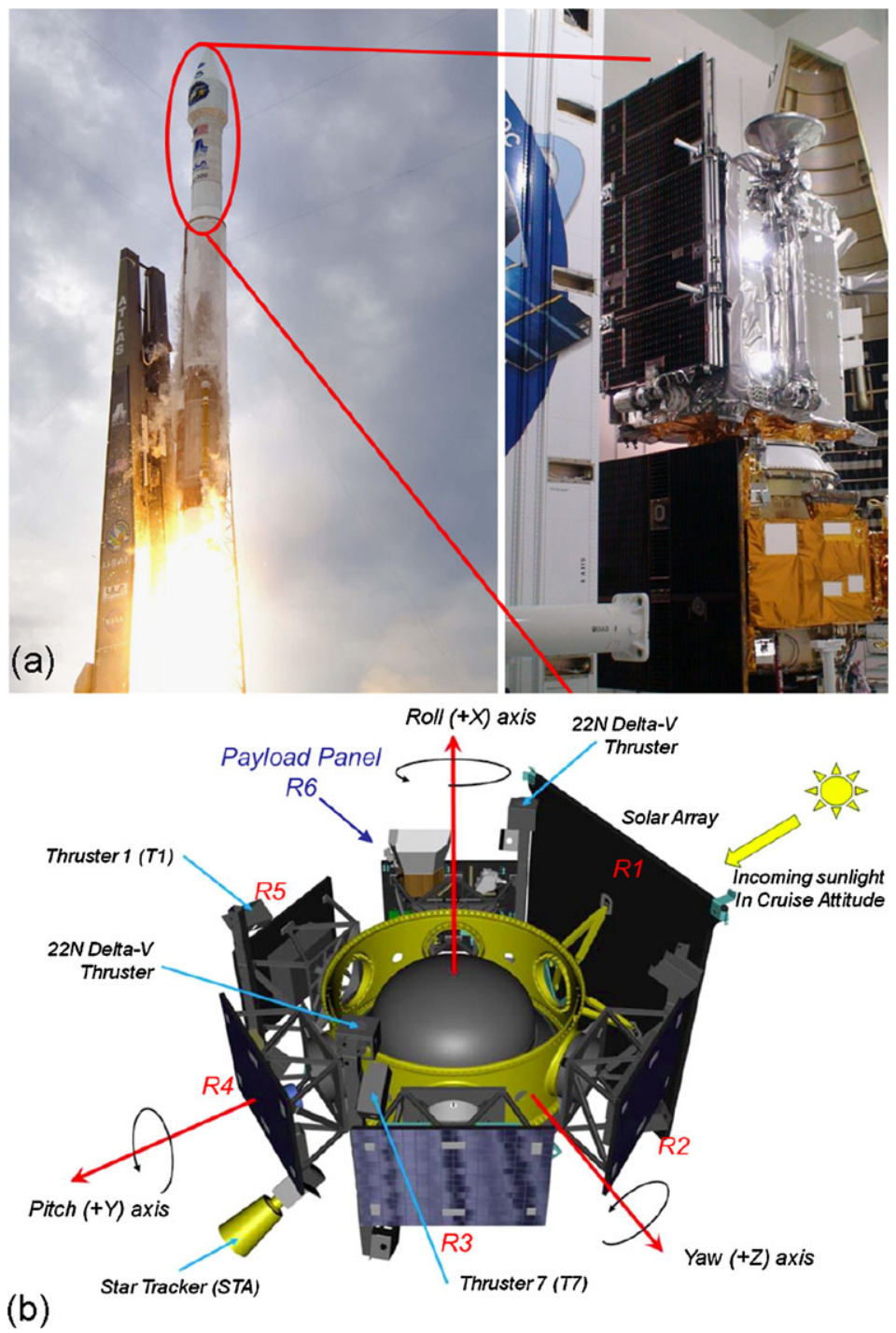

Fig. 1 (a) LCROSS in its launch configuration between the LRO and the Atlas V Centaur Rocket. LRO and LCROSS launched successfully on June 18, 2010. The right image shows the inside of the Atlas V payload fairing with LCROSS at the bottom in gold and LRO on top in silver. LRO separated away forty-five minutes after launch, but LCROSS stayed connected to the Atlas V Centaur upperstage for its mission. (b) The LCROSS payload's location on the S-S/C and the defined spacecraft axes (roll, pitch, yaw). The $-X$ spacecraft axis was the launch axis and the nominal nadir pointing axis during the final descent to the Moon at end of mission. The Solar Array was aligned along the $-Y$ spacecraft axis. During cruise phase, this axis was directed towards the Sun. The S-S/C backbone was an ESPA ring. The S-S/C height was 2 meters tall. The S-S/C basic structure was 2.6 meters in diameter. From "omni $-\mathrm{Z}$ " to "omni $+\mathrm{Z}$ " antennae, the $\mathrm{S}-\mathrm{S} / \mathrm{C}$ was 3.3 meters wide. The six panels attached to the ESPA ring were numbered clockwise with the Solar Array as R1, R2, R3, R4, and R5 structurally supported the batteries, the power control electronics, the attitude \& communications electronics, and the command \& data handling electronics, respectively. The LCROSS payload was located on the R6 panel. A propellant tank sat within the ESPA ring center. Thrusters, four communication antennas (two omni, two medium gain), and a single star tracker, are located as shown. A wiring harness (not shown in this schematic) connected all the electrical subsystems 
Table 1 LCROSS Level 3 science requirements for the payload design

Requirement Description

4.1.1 LCROSS shall be capable of detecting the total water in the sunlit ejecta cloud at concentrations of as little as $0.5 \%$ by mass.

4.1.2 LCROSS shall be capable of measuring the concentrations of detected total water (by mass) in the sunlit ejecta cloud with a 'factor of 3' measurement accuracy.

4.1.3 LCROSS shall be capable of detecting hydrogen-bearing compounds in the sunlit ejecta cloud at concentrations greater than $10 \%$ by mass.

4.1.4 LCROSS shall be capable of measuring total sunlit ejecta cloud mass with a 'factor of 2' measurement accuracy.

4.1.5 LCROSS shall be capable of measuring the bulk mineralogy in the sunlit eject cloud.

4.1.6 LCROSS shall be capable of measuring the mean grain size of the sunlit ejecta cloud for particles sizes between 10 and 100 microns.

4.1.7 LCROSS shall be capable of observing impact target location and the sunlit ejecta cloud with at least $0.1 \mathrm{~Hz}$ frequency from Centaur impact minus 5 minutes until Centaur impact plus 3 minutes. (Note: Assumes LCROSS S-SC trails Centaur by 4 minutes.) The LCROSS S-SC is not required to maintain observation of the Centaur impact target location during the minute preceding S-SC impact of the lunar surface).

4.1.8 LCROSS shall be capable of imaging a minimum area of $100 \mathrm{~km} \times 100 \mathrm{~km}$ at time of impact with at least 500 meter resolution.

Table 2 LCROSS measurement goals and requirements trace to science instrument

Goals and requirements

\begin{tabular}{lllllllllll}
\hline Keyword & $\begin{array}{l}\text { Total } \\
\text { water }\end{array}$ & $\begin{array}{l}\text { Total } \\
\text { ice }\end{array}$ & $\begin{array}{l}\text { Total } \\
\text { vapor }\end{array}$ & Organics & $\begin{array}{l}\text { Hydrated } \\
\text { minerals }\end{array}$ & $\begin{array}{l}\text { Curtain } \\
\text { mor- } \\
\text { phology }\end{array}$ & $\begin{array}{l}\text { Thermal } \\
\text { evolu- } \\
\text { tion }\end{array}$ & $\begin{array}{l}\text { Miner- } \\
\text { alogy }\end{array}$ & $\begin{array}{l}\text { Particle } \\
\text { size }\end{array}$ & $\begin{array}{l}\text { Target } \\
\text { proper- } \\
\text { ties }\end{array}$ \\
\hline Goal & 1,2 & 1,2 & 1,2 & 2 & 2 & 2 & 3 & 3 & 3 & 4 \\
\hline Req't & $4.1 .1-$ & $4.1 .1-$ & $4.1 .1-$ & 4.1 .3 & 4.1 .3 & $\begin{array}{l}4.1 .4, \\
4.1 .7-\end{array}$ & 4.1 .4 & 4.1 .5 & 4.1 .6 & 4.1 .7 \\
& 4.1 .4 & 4.1 .3 & 4.1 .3 & & & 4.1 .8 & & & & \\
\end{tabular}

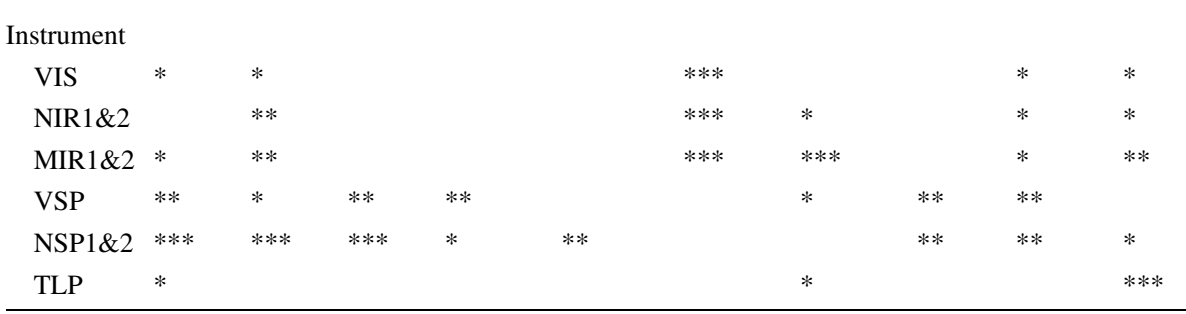

Goal numbers are described in text. The science requirements are defined in Table 1. Key: *** Direct/Strong: Very direct measure to goal(s) with little modeling or assumption/highly sensitive; ** Indirect/Strong: Indirect measure to goal(s) but removed by several steps/highly sensitive; * Indirect/Weak: Indirect measure to goal(s), but removed by several steps/moderately sensitive. Instrument acronyms are defined in Sect. 3

the near-infrared spectrometers (NSP1\&2) provided the most direct measurement for the total water content in the ejecta curtain, and as such, these instruments were given the greatest amount of test scrutiny.

The trace between project Level 3 requirements and the instruments, indicating both measurement technique and instrument priority is shown in Table 3. Assignment of instrument 
Table 3 LCROSS Level 3 science requirements and science instrument priority trace

\begin{tabular}{|c|c|c|c|c|c|c|c|c|}
\hline \multirow[t]{2}{*}{ Instrument } & \multicolumn{8}{|c|}{ LCROSS project Level 3 science requirements } \\
\hline & 4.1 .1 & 4.1 .2 & 4.1 .3 & 4.1 .4 & 4.1 .5 & 4.1 .6 & 4.1 .7 & 4.1.8 \\
\hline \multirow{4}{*}{$\begin{array}{l}\text { Visible } \\
\text { Camera (VIS) } \\
\text { NIR Cameras } \\
\text { (NIR1/NIR2) }\end{array}$} & $*$ & $*$ & & $* * *$ & & $*$ & $* * *$ & $* * *$ \\
\hline & A & A & & $\mathrm{P}$ & & A & $\mathrm{P}$ & $\mathrm{P}$ \\
\hline & & $*$ & $* *$ & $* * *$ & $*$ & $*$ & $* * *$ & $* * *$ \\
\hline & & A & A & $\mathrm{S}$ & A & A & $\mathrm{S}$ & $\mathrm{S}$ \\
\hline \multirow{2}{*}{$\begin{array}{l}\text { MIR Cameras } \\
\text { (MIR1/MIR2) }\end{array}$} & & $*$ & $* *$ & $* * *$ & $*$ & $*$ & $* * *$ & $* * *$ \\
\hline & & $\mathrm{S}$ & A & $\mathrm{S}$ & A & $\mathrm{S}$ & $\mathrm{S}$ & $\mathrm{S}$ \\
\hline \multirow{2}{*}{$\begin{array}{l}\text { UV-visible Spect. } \\
\text { (VSP) }\end{array}$} & $* *$ & $* *$ & $* *$ & $* *$ & $* * *$ & $* *$ & $* * *$ & \\
\hline & A & $\mathrm{S}$ & $\mathrm{S}$ & A & S & S & A & \\
\hline NIR & $* * *$ & $* * *$ & $* * *$ & $* *$ & $* * *$ & $* *$ & $* * *$ & \\
\hline $\begin{array}{l}\text { Spectrometers } \\
\text { (NSP1/NSP2) }\end{array}$ & $\mathrm{P}$ & $\mathrm{P}$ & $\mathrm{P}$ & A & $\mathrm{P}$ & $\mathrm{P}$ & A & \\
\hline \multirow{2}{*}{$\begin{array}{l}\text { Total Lum. Phtm. } \\
\text { (TLP) }\end{array}$} & $*$ & & & & & & $* *$ & \\
\hline & A & & & & & & A & \\
\hline
\end{tabular}

Key: ***, **, * Same as in Table 1 . The Level 3 science requirements are defined in Table 1. (P) (Primary Method): Required to full meet the Level 3 requirements. (S) (Secondary Method): Could meet Level 3 requirements. (A): Addresses and/or partially meets Level 3 requirements

priority affected instrument design, test requirements, instrument sparing strategy, flight sequence development, and the payload failure modes and effects analysis approach (see Sect. 4). For example, imaging of the ejecta cloud was primarily to support requirements associated with estimating the total ejecta mass from which an estimate of the water concentration could be made. The approach to making this measurement used measurements of the sunlight scattering off of the ejecta cloud from the spectrometers and the geometry of the cloud from the cameras. This approach did not drive requirements for absolute radiometric calibration on the cameras and thus radiometric calibration of the cameras was minimal.

Likewise, the approach to measuring water concentration was differential absorption. Since pre-impact reference spectra of the scene could be made, post-impact spectra could be ratioed to the pre-impact reference to reveal relative differences in pre- and post-impact spectra, or ratioed to the observed pre-impact solar spectrum in the case of the solar-viewing near infrared spectrometer (NSP2). These differences were used to compute to the total amount of water absorption through linear fitting and radiative transfer calculations (Colaprete et al. 2010). This approach was selected as it did not require rigorous absolute radiometric calibration of the near infrared spectrometers.

The LCROSS approach in its definition of the mission measurement goals (at the Program level) and the generation of requirements at the Project level was atypical of other non-Class D science missions. Project level measurement requirements worked within a constrained set of capabilities allowed by the budget and schedule. Where requirements could be minimized or dropped, they were. A standing mantra for the LCROSS Science and Payload team was: "Be just good enough". As such, many of the usual tests and calibrations associated with Class $\mathrm{C}$ and above missions were not carried out for the LCROSS Payload.

LCROSS was a capability-driven mission. That is, to remain inside the constraints of cost and schedule, COTS instruments needed to be considered, rather than the development of new instruments. This fact was most acute on LCROSS as the entire payload was not required for minimum mission success. That is, if an instrument appeared to be in trouble with respect to cost or schedule, it could be descoped outright. The processes for instrument 
selection began with the mission's science measurement requirements (see Table 1) and a survey of COTS instruments that most closely met those requirements with no or minimal modification. In each case the COTS instrument was evaluated for its ability to meet flight environmental criteria, cost and schedule. Also carefully scrutinized was whether the instrument could meet other engineering requirements (e.g., RS422 interface). Instruments that were deemed more important to the success of the overall measurement goals (see Tables 2 and 3), for example the spectrometers, were given added resources, in terms of financial and personnel support, as well as evaluation and calibration. This was necessarily done at the expense of other instruments that had a lower level of priority (e.g., the cameras). At times, measurement requirements were reevaluated if it became clear that the requirement could not be met given available resources. In these instances, any change in a requirement was presented to the Program Office for approval.

Prior to selection of specific instrument vendors, the LCROSS Payload Team worked with the vendors to define any necessary modifications from the COTS instrument. At times, other mission requirements were modified to accommodate the limits of using COTS instruments. In some cases, like the fore-optics to the nadir spectrometers, there was no COTSequivalent. The design and procurement of these also started first from the science measurement requirements, but needed to be compliant with the COTS instruments' interfaces and meet the LCROSS payload mass and volume requirements. Arriving at the $1^{\circ}$ diameter FOV for the two nadir spectrometers was an interesting trade. Technically, the final optical solution needed to match the required numerical apertures for the COTS spectrometers, while maximizing the aperture to meet the measurement sensitivity requirements. Scientifically, that same FOV was required to match a footprint of $\sim 10 \mathrm{~km}$ (the predicted diameter of the ejecta cloud) from an altitude of $600 \mathrm{~km}$. A fixed $1^{\circ}$ diameter FOV met the optical interface requirements and volume restraints, but in order to have this FOV subtend the ejecta cloud and meet the sensitivity, the project had to adjust the S-S/C separation to four minutes. This solution maximized the throughput (factoring in changes in ejecta cloud scattering flux densities and aperture fill factor). This, in turn, had to be traded against the S-S/C pointing capability to ensure the spectrometers $1^{\circ}$ diameter FOV stayed on target.

The LCROSS project also solicited ground- and orbital-based measurements of the ejecta plume in order to provide ancillary information from those platforms' unique viewpoints. The observation campaign approach and measurements types are described in Heldmann et al. (2011). In addition, prior to impact, the LCROSS science team worked with the LRO Project team to obtain early LRO observations with LEND, LOLA and Diviner of the lunar South Pole to aid in target selection (Mitrofanov et al. 2010; Paige et al. 2010b). On impact day, LRO's Diviner and LAMP instruments observed the ejecta plume. Their data summaries are described in Hayne et al. (2010) and Gladstone et al. (2010).

\section{Payload Description}

The LCROSS payload consisted of nine science instruments, their supporting electrical, mechanical and optical harnesses, a central data handling unit (DHU), and thermal hardware (heaters, thermistors, and thermostats). Eight of the nine science instruments were configured with their apertures aligned along the spacecraft $+X$ direction and were accommodated in the Payload Observation Deck (POD). This axis defined the nadir-view to the Moon during terminal approach. The ninth instrument, the solar near infrared spectrometer (NSP2), had its aperture orientated a few degrees from the spacecraft $-Z$ axis, directed towards the Sun during terminal approach. The payload was located on the inboard side of the LCROSS 
spacecraft R6 Radiator Panel (Fig. 1b). A description of the LCROSS spacecraft design can be found in Segura et al. (2010).

All nine science instruments successfully collected data during all in-flight calibration campaigns, and most importantly, during the final descent to the lunar surface on October 9, 2009, after 112 days in space. The LCROSS payload science instruments' orientation and location in the POD and on the R6 panel are shown in Figs. 2a and 2b, respectively. The individual instruments are shown in Fig. 3 and are described below.

\subsection{Visible Camera}

The LCROSS visible camera (VIS) (Fig. 3a) was a ruggedized analog video camera from the RocketCam ${ }^{\mathrm{TM}}$ ED360513 camera family developed by Ecliptic Enterprises, Corporation. The unit consisted of a COTS Sony camera module and lens and a ruggedized mount. The focal plane was a $1 / 2^{\prime \prime}$ color CCD. The visible camera's $12 \mathrm{~mm}, \mathrm{f} / 1.2$ lens provided a $30.1^{\circ}[\mathrm{H}] \times 22.8^{\circ}[\mathrm{V}]\left(37.8^{\circ}\right.$ [Diagonal]) field-of-view (FOV). Requiring 2.9 Watts, the camera was operated at $30 \mathrm{~Hz}$ and created interlaced, NTSC-formatted images that were digitized as $720 \times 486$ pixel images (in two $720 \times 243$ bit fields). Each pixel was captured as 24-bit RGB ( 8 bits per color channel). The digitized images were then compressed by a proprietary lossy, wavelet-based compression algorithm performed by an Analog Devices 611 Video Codec configured to produce an average 20:1 compression ratio before transmission to the ground. The measured pixel iFOV was $0.043^{\circ}[\mathrm{H}] \times 0.047^{\circ}[\mathrm{V}]$.

In its flight configuration, the visible camera was fixed at an auto-gain/white balance setting with auto-exposure. Prior to the LCROSS launch, this camera type had flown on over 40 missions, the majority being of extremely short duration (e.g., $<30$ minutes as context imagers on rockets or space shuttle external tanks) (Ridenoure 2004). Two visible cameras were attached to the LRO/LCROSS Atlas V (AV020) vehicle and provided launch and payload fairing ejection images, but these were not part of the LCROSS payload.

\subsection{Near Infrared Cameras}

The two LCROSS near infrared (NIR) $(0.9-1.7 \mu \mathrm{m})$ cameras (Figs. 3b and 3c) were Goodrich Sensors Unlimited InGaAs model SU320-KTX fitted with a non-IR optimized CCTV glass lens. Their $25 \mathrm{~mm}, \mathrm{f} / 1.4$ lenses provided a $28.7^{\circ}[\mathrm{H}] \times 21.7^{\circ}[\mathrm{V}]\left(36.0^{\circ}[\right.$ Diagonal]) FOV. In the LCROSS payload design, to simplify the DHU interface to meet a short development schedule, these cameras were operated in 8-bit analog mode, although the cameras themselves were capable of 12-bit digital resolution. These cameras had $320 \times 240$ pixel format, but were repackaged to a $720 \times 486$ pixel NTSC format via the DHU. This format is identical to the description provided in Sect. 3.1 for the visible camera, but because the NIR cameras provided grayscale images, the three RGB channels were identical except for noise introduced in the conversion to and from NTSC. Their measured pixel iFOV was $0.040^{\circ}[\mathrm{H}] \times 0.045^{\circ}[\mathrm{V}]$.

One of the NIR cameras (NIR1) contained a long pass filter $(\lambda>1.4 \mu \mathrm{m})$. Each camera's peak power during operation was 1.6 Watts. Both gain and exposure times were configurable by commands. The cameras were capable of integration times between 0.11 and $16.24 \mathrm{~ms}$. The ability to change exposure time addressed large dynamic ranges seen in the LCROSS targets (see Sect. 6). Early in the payload development, a trade study on the lens choice was performed. Due to budget restraints and their lower priority (see Table 3), IRoptimized lenses were not purchased for these cameras, even though they did exist. The vendor-provided CCTV glass lens was used. These cameras had no space-qualification testing prior to the LCROSS mission. Therefore, they underwent full ground-based space qualification testing before flight. 

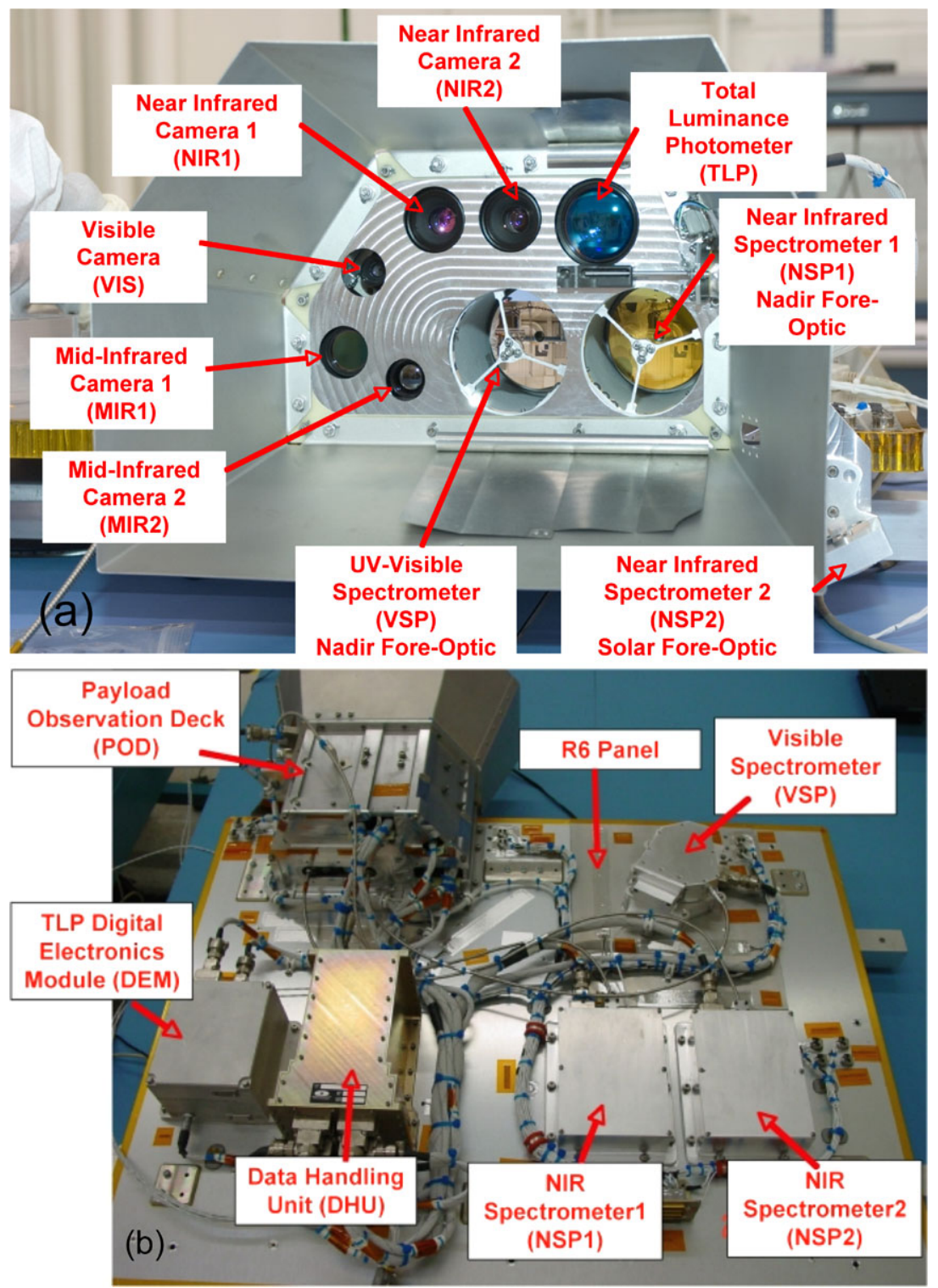

Fig. 2 The LCROSS payload on the S-S/C R6 Panel. (a) A view of the apertures from the payload observation deck (POD) viewpoint. The five cameras, the TLP, and the fore-optics for the VSP and NSP1 are seen from this viewpoint. At the time of final descent the POD's apertures were directed towards the impact site, along the $-X$ spacecraft axis. (b) The location of the POD and the identification of the instruments not enclosed in the POD are shown. The R6 Panel measures $101.6 \times 77.5 \mathrm{~cm}(40 \times 30.5$ inches $)$. The POD Shade extents the dimension along the $-X$ axis to $99.8 \mathrm{~cm}$ (39.3 inches)

\subsection{Thermal Cameras}

The two mid-infrared $(6.0-13.5 \mu \mathrm{m})$ uncooled VoX bolometer cameras were procured from different vendors, one MIRIC ${ }^{\circledR}$ TB2-30 camera from Thermoteknix Ltd. (MIR1) 

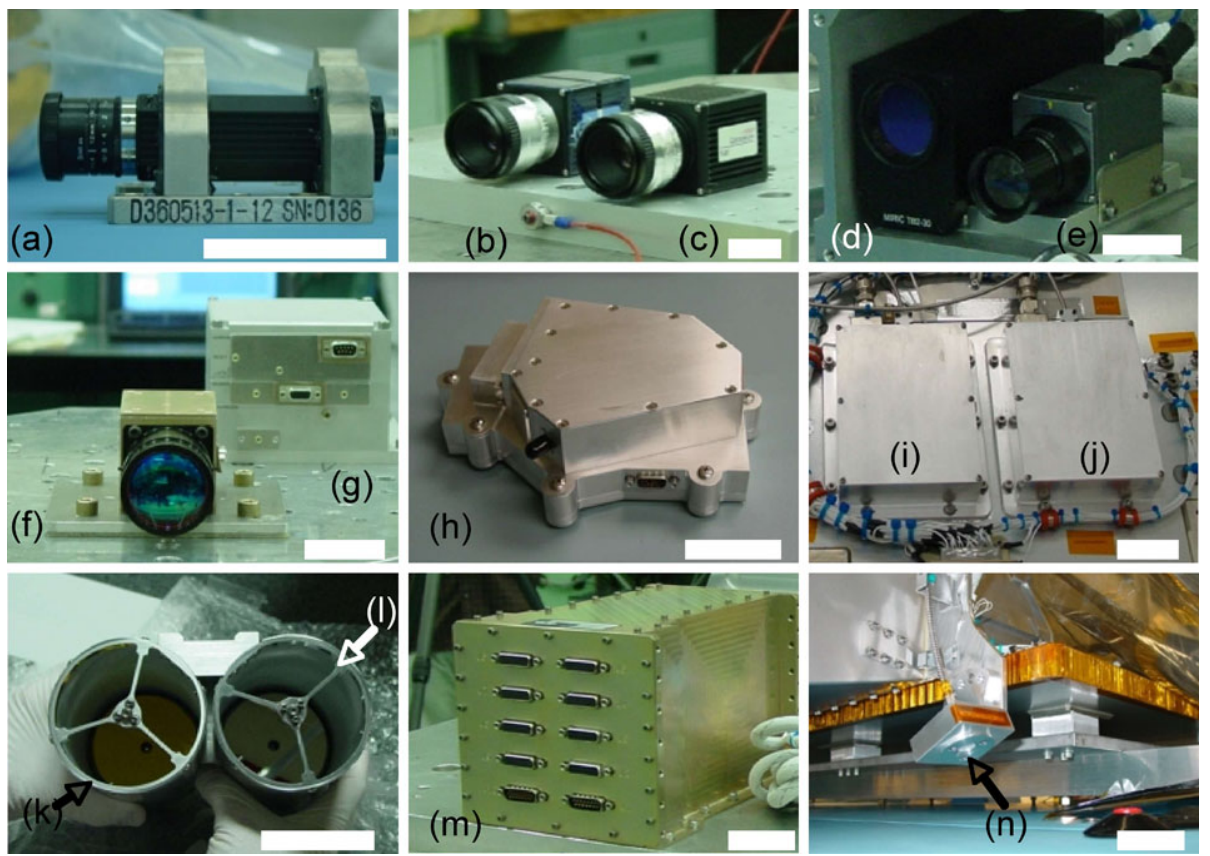

Fig. 3 The LCROSS payload science instruments and optics. The panels are labeled (a) visible camera (VIS), (b) near infrared camera \#1 (NIR1), (c) near infrared camera \#2 (NIR2), (d) mid-infrared camera \#1 (MIR1), (e) mid-infrared camera \#2 (MIR2), (f) Total Luminance Photometer (TLP) Sensor Electronics Module (SEM), (g) TLP Digital Electronics Module (DEM), (h) UV-visible spectrometer (VSP), (i) near-infrared spectrometer \#1 (NSP1), (j) near-infrared spectrometer \#2 (NSP2), $1^{\circ}$ diameter FOV (k) VSP fore-optics and (I) NSP1 fore-optics, (m) Data Handling Unit (DHU), and (n) $130^{\circ}$ diameter FOV NSP2 fore-optics. Descriptions of each instrument are found in the text. The horizontal scale bar in the lower right of each image represents $5.08 \mathrm{~cm}$ ( 2 inches)

(Fig. 3d) and one ThermoVision Micron camera from Flir Systems/Indigo Operations (MIR2) (Fig. 3e). The payload design needed one MIR camera. The use of Flir as the vendor for MIR2 was chosen as a mitigation against cost and schedule challenges in procuring a second MIR from Thermoteknix. Each camera had a $30 \mathrm{~mm}, \mathrm{f} / 1.6$ lens that provided a $15.0^{\circ}[\mathrm{H}] \times 11.0^{\circ}[\mathrm{V}]\left(18.6^{\circ}\right.$ [Diagonal] $)$ FOV. Each camera's uncooled microbolometer focal plane sensor had a $164 \times 128$ pixel format and was digitized at 14-bit resolution. The measured pixel iFOV was $0.097^{\circ}[\mathrm{H}] \times 0.097^{\circ}[\mathrm{V}]$.

One MIR camera (MIR1) contained a band pass filter $(6-10 \mu \mathrm{m})$ and had been backfilled with dry Argon gas to prevent degradation from humidity for its typical terrestrial applications. This configuration would prove to be essential for good performance in vacuum compared to the other unit (MIR2) (see Sect. 6). Each camera's peak power during operation was 1.3 Watts. Internal to each MIR camera was an instrumented-motorized shutter that provided reference measurements. This reference was not used for temperature calibration, only for indicating when the camera needed to flat-field correct its response during normal operation. For LCROSS, the cameras were set to high gain mode to allow for best sensitivity to scenes $-20^{\circ} \mathrm{C}<T<+150^{\circ} \mathrm{C}$. These cameras also possessed a low gain mode, optimized for scenes $+150^{\circ} \mathrm{C}<T<+500^{\circ} \mathrm{C}$. Low gain was not used in flight except for diagnostic purposes. Prior to LCROSS, the Thermoteknix camera had limited space qualification test- 
ing, only the characterization of solid rocket motor debris during an Aegis ballistic missile defense test (Gauthier et al. 2006). The Flir camera had no prior space qualification testing.

\subsection{Flash Photometer}

The Total Luminance Photometer (TLP) provided visible light (400-1000 nm) intensity data at a $1000 \mathrm{~Hz}$ sampling rate. This instrument was composed of a Sensor Electronics Module (SEM) (Fig. 3f) that contained the optics, sensor, and signal filtering, and a Digital Electronics Module (DEM) (Fig. 3g) that converted the analog sensor signal to a digital output. The SEM was designed at NASA Ames. The SEM's sensor element was an uncooled Advanced Photonix, Inc. avalanche photodiode (APD) module SD 394-70-7x-661 packaged in a dryair sensor container that could operate in a vacuum. In its operation, the APD amplified the received optical signal and outputted a signal voltage proportional to the pulse shape of the observed flash signal. The resulting signal voltage passed through a high-pass filter to remove the background light level, was further amplified $(\times 100)$, and finally passed through a low-pass filter to remove the out-of-band high frequency noise. The processed signal contained only the flash signal and any remaining noise within the pass-band of $0.014 \mathrm{~Hz}$ to $406 \mathrm{~Hz}$.

The SEM optics delivered an unobstructed $\sim 10^{\circ}$ diameter FOV. The DEM was a commercial package. The SEM and DEM's peak power during operation were 2.5 Watts and 12.0 Watts, respectively. Neither part had prior space qualification testing. This instrument was constructed early in the payload development schedule and underwent extensive iterative development to make its design robust (see Sect. 4).

\subsection{Ultraviolet-visible Spectrometer}

The LCROSS UV-visible spectrometer (VSP) (Fig. 3h) was a modified-commercial QE65000 model from Ocean Optics, Ltd. Its core design was adapted for space use as part of the Mars Science Laboratory ChemCam instrument (Saccoccio et al. 2009). For LCROSS, changes to the VSP included repackaging of the spectrometer board and detector. A significant change was in how the instrument managed its thermal state. The COTS instrument used a fan, while the LCROSS VSP employed a heat sink that was thermal coupled via the instrument housing to the payload panel and radiator. From an electrical and optical standpoint no other changes were made. Additional aluminum was used to provide radiation shielding, but no use of radiation hardened or tolerant parts were used.

In the LCROSS configuration, a single 0.11 NA (numerical aperture), $75 \mathrm{~cm}$ length, 600 micron core-diameter Fiberguide Industries UV/Vis glass fiber fed a 25 micron $\times 1 \mathrm{~mm}$ entrance slit. A $25.4 \mathrm{~mm}$ (1-inch) f/4 optical cross Czerny-Turner spectrometer (grating 600 lines $/ \mathrm{mm}$, blazed at $350 \mathrm{~nm}$ ) with an oversized camera mirror delivered a 263-650 nm spectrum onto a $1044 \times 64$ pixel Hamamatsu CCD detector. The spectrometer internally row co-added the pixels to deliver a 16-bit, $1 \times 1044$ pixel spectrum to the LCROSS DHU electronics for downlink. The pixels contained both spectral and housekeeping dark reference data, the latter was used for calibration (see Sect. 6.3.1 for a description and layout in Fig. 10).

The other fiber end attached to a specifically designed fore-optics unit in the POD. This fore-optics unit (Fig. 31), built by Aurora Design \& Technologies, Inc., was a fixed twomirror and one lens system designed to provide a one degree diameter FOV. Spectral resolution, $R \sim 300-850$, was measured for the VSP (see Sect. 6.3.1 for line profile full width half-maximum (FWHM) values). The VSP's CCD detector was cooled by an internal thermoelectric cooler (TEC). The spectrometer's power during operation was 4.8 Watts (TEC 
off) but reached 11.8 Watts (TEC setting $-10^{\circ} \mathrm{C}$ for $+20^{\circ} \mathrm{C}$ operating temperature). The TEC setting $-10^{\circ} \mathrm{C}$ was used for all flight data. Integration time (between $8 \mathrm{~ms}$ and $65.5 \mathrm{~s}$ ) was configurable by software command.

Two operation modes were developed for the LCROSS UV-visible spectrometer: (1) single mode where a single spectrum was acquired with the requested integration time; and (2) bracket mode where three spectral acquisitions were defined by a base integration time value and a multiplier. The former was divided or multiplied by the latter to provide a shorter or longer exposure time, respectively, which, with the base spectrum, formed a triplet spectra with minimized ( $<7 \mathrm{~ms}$ ) dead time gaps between spectra. Although parts of this spectrometer had undergone spaceflight design, the packaging was unique to LCROSS and therefore had to be fully re-qualified.

\subsection{Near Infrared Spectrometers}

The LCROSS payload contained two near-infrared spectrometers (NSP) manufactured by Polychromix, a company whose primary spectrometer line is designed for material analysis and chemical sensing. Their electronics units were identical, but had different fore-optics designs. NSP1 (Fig. 3i) was also known as the nadir NSP because its fore-optics unit (Fig. 3k) was located inside the POD. NSP1 fore-optics was a fixed two-mirror and one lens system designed to provide a one degree diameter FOV, co-aligned with the UV-visible spectrometer fore-optics to within $0.1^{\circ}$ (see Sect. 6.2). NSP2 (Fig. 3j), the solar-viewer or occultation NSP, had a diffuser (Fig. 3n) that provided a $\sim 130^{\circ}$ FOV aligned along the axis predicted for the Sun during the terminal phase of the mission. Both fore-optics were designed by Aurora Design \& Technologies, Inc. and had no prior space qualification.

The near infrared spectrometers each took a single NA $=0.22600$-micron core-diameter low-OH Fiberguide Industries glass fiber as an input instead of a slit. NSP1 and NSP2 fiber lengths were $75 \mathrm{~cm}$ and $1.45 \mathrm{~m}$, respectively. Both spectrometers provided 1.20-2.45 $\mu \mathrm{m}$ spectral coverage at a nominal resolution $0.035 \mu \mathrm{m} /$ pixel. Spectral resolution, $R \sim 37-65$ and $R \sim 36-77$, were measured for NSP1 and NSP2, respectively, for Hadamard mode (see Sect. 6.3.2 for FWHM values). This specification was sufficient for water absorption profile measurements. The instrument is capable of higher spectral resolution using smaller input fiber diameters at the expense of lower throughput. Internal aperturing of the spectrometer limited the lowest resolution. Each spectrometer contained a single TEC-cooled InGaAs sensor element. A spectrum was created using an innovative electronically-tunable MEMS device that spatially masked the dispersed spectrum within the instrument (Day et al. 2005). This mask series underwent a digital transform on the ground to recreate the spectral information. The peak power for each NSP was 2.5 Watts.

Both near infrared spectrometers had three operation modes: Flash, Hadamard, and Diagnostic. Flash mode provided spectral samples in five discrete bands (1.39-1.50, 1.56$1.66,1.82-1.93,1.98-2.09,2.28-2.38 \mu \mathrm{m}$ ) at $72 \mathrm{~Hz}$ sampling rate (see Sect. 6.3.2). It was used for calibration during limb crossings of the Moon and Earth and during the period expected for the Centaur's impact flash (see Sect. 5.1). Hadamard mode, the primary mode, provided a complete $1.20-2.45 \mu \mathrm{m}$ spectrum at $1.7 \mathrm{~Hz}$. Diagnostic mode provided information at power-on in case the instrument failed to work properly. The near infrared spectrometers had the highest priority (see Table 3 ). They also had no prior space heritage and were tested early and iterated to robust their design (see Sect. 4).

\subsection{Data Handling Unit (DHU)}

The LCROSS DHU (Fig. 3m) was an instrument controller and telemetry formatting system based on Ecliptic Enterprises Corporation's RocketCam Digital Video System (Ridenoure 
2004). The DHU powered, sent commands to and formatted data from all nine instruments. The DHU received $32 \mathrm{~V}$ from the S-S/C electrical bus and converted it into $3.3 \mathrm{~V}$ for DHU internal circuitry and $5 \mathrm{~V}$ and $12 \mathrm{~V}$ for various instruments. The DHU also passed $32 \mathrm{~V}$ directly to the UV-visible and near infrared spectrometers. The DHU itself used 13.5 Watts (peak). DHU flight software was developed by Ecliptic with $70 \%$ heritage from previous missions and $30 \%$ custom-developed for LCROSS to Ames' specifications.

The visible light camera received power only, but the other instruments received power and operational mode commands from the DHU. The DHU, in turn, received commands from the S-S/C flight computer. S-S/C commands could be passed through to the instruments or initiate one of ten instrument command sequences stored in DHU Non-Volatile Memory (NVM). Both styles of commanding were used on the LCROSS flight mission, but most of the payload operations used command sequences stored in the DHU (see Sect. 5). These sequences ranged from 10 to 450 commands in length.

The DHU also captured science data from the instruments, formatted this data as CCSDS packets and frames, and sent it via SpaceWire to the LCROSS transponder for downlink in real-time. No onboard storage was required or provided for instrument data. The DHU also applied timestamps to all instrument data and provided housekeeping telemetry describing when science data was dropped or corrupted due to bandwidth limitations or performance issues. The DHU flight software that performed these functions was $\sim 10 \mathrm{k}$ lines of code, $70 \%$ heritage, $30 \%$ custom-developed code for LCROSS. The new code was primarily related to instrument interfaces, although there were small changes in other modules.

The DHU could operate almost completely independently from the rest of the spacecraft, requiring only power, commands and a place to send fully-formatted telemetry. This drastically simplified the payload-to-spacecraft interface and allowed payload integration and testing to occur prior to and physically separate from spacecraft integration. This separation, in turn, was instrumental in allowing the flight control team to build up a lot of experience operating the integrated instrument suite before launch, which was important because there were many subtle interactions, between the DHU and individual instruments and between instruments themselves. This advantage continued during the flight, as the ground spacecraft and payload simulators could be operated in either connected or disconnected modes, reducing competition for simulator time during periods of intense activity.

\subsection{Thermal Control}

The LCROSS payload's thermal control was maintained via localized R6 radiating surfaces and multi-layered insulation (MLI). MLI covered all external surfaces except the payload optics and radiators. Silverized Teflon tape was used on all radiating surfaces. Heaters were sized during design, and software-controlled during flight, to maintain payload temperature for the bakeout, survival, and operational modes of the mission, and to allow for changes to on/off control setpoints. The thermal heater design had primary and redundant circuits. Finally, a thermostat was connected to each heater circuit for over-temperature protection in case software control of the heater circuit failed.

\section{Class D Payload Development Program}

LCROSS was defined per NASA's NPR 8705.4, "Risk Classification for NASA Payloads" as a NASA Class D mission, the most risk-tolerant category (NPR 8705.4 2008). Technical risk played a significant role, managed rigorously and openly, throughout the mission 
design, development and operation. LCROSS was firmly cost-capped, under threat of cancellation if costs were exceeded. LCROSS was also schedule-constrained since it had to make the LRO launch date. As a result, the LCROSS project was allowed to tailor performance requirements or take on additional risk if necessary in order to meet the cost and schedule constraints (Andrews 2010).

Keeping technical risk in check meant that the LCROSS mission was not about pushing the limits of technology and performance. The LCROSS mission was about doing as much as possible within the existing system capabilities. This directly affected the science payload design, development, and testing approach.

The LCROSS payload subsystem was also cost-capped (to \$1M) and scheduleconstrained to a nine-month qualification period. To fit within these constraints, low-cost, ruggedized, commercial off-the-shelf (COTS) components were heavily emphasized. Some LCROSS payload science instruments had little or no space qualification testing. For those instruments, to mitigate risk, the LCROSS payload test program stressed early verification testing of Engineering Test Units (ETUs) that, for the most part were identical in form and function to the flight versions. These early tests were development tests at qualification and protoqualification (protoflight) levels. Here, the test environment was more severe than expected during the operational life of the hardware (e.g., temperatures were more extreme, level of vibration induced was higher than expected for launch, number of thermal cycles was higher). The purpose of this testing verified workmanship, material quality and structural integrity of the design. The tests were shared between NASA/ARC and the instrument vendors to alleviate schedule burden and promote rapid turn-around for flight unit development. This proved to be a successful paradigm to increase the robustness of this Class D payload over the course of a few months.

The flight instruments underwent a tailored space qualification program at acceptance levels to simulate launch and conditions in space, with sufficient mission assurance oversight to maintain safety and adherence to key technical performance requirements that flowed from the Level 1 \& 2 mission requirements. The payload test program used Goddard's General Environmental Verification Standard (GSFC-STD-7000 2005). tailored for an unpowered launch and a low number $(<10)$ of power cycles in the space environment. For example, an EMI/EMC compatibility test between the payload electrical interface and the LCROSS $\mathrm{S}-\mathrm{S} / \mathrm{C}$ was required rather than a complete EMI/EMC test on each payload unit, as would typically be required. This was an acceptable risk since careful EMI/EMC shielding and filters were implemented in each instrument design at the vendor level and reviewed by the LCROSS system engineers.

The development and flight test matrix designed and used for the LCROSS payload is shown in Table 4. When possible, tests were performed at the unit level. Schedule constraints and limited personnel and resources sometimes forced testing at the sub-assembly level. Although this could have led to more complex anomaly resolution, emphasis was placed on individual instrument performance when possible at higher assembly levels.

The actual accumulated powered test hours per instrument is summarized in Table 5. The flight instruments averaged $40 \times$ more hours in pre-flight testing than in operation on orbit. However, most instruments only achieved an average of $0.5 \times$ the hours in pre-flight vacuum testing than in operation, with the exception of the DHU and NSPs which were tested in vacuum for $1.5 \times$ their operations time. The payload was given a requirement of 40 hours total powered testing, but this was not specified to be at vacuum. Such a requirement is typical for short duration Department of Defense (DOD) mission applications (e.g., see MIL-HDBK-343 1986).

The LCROSS payload integration and test (I\&T) approach grew from a strict and limited test schedule and did deviate from previous standard testing practices. A comparison of the 
Table 4 LCROSS payload test matrix for both development and flight units

\begin{tabular}{|c|c|c|c|c|c|c|c|c|}
\hline Instrument & $\begin{array}{l}\text { Assembly } \\
\text { level }^{\mathrm{a}}\end{array}$ & $\begin{array}{l}\text { Func- } \\
\text { tional }\end{array}$ & $\begin{array}{l}\text { Bake- } \\
\text { out }\end{array}$ & $\begin{array}{l}\text { Therm. } \\
\text { cycle }\end{array}$ & $\begin{array}{l}\text { Therm. } \\
\text { vac. }\end{array}$ & EMI/EMC & $\begin{array}{l}\text { Rand. } \\
\text { vibe }\end{array}$ & $\begin{array}{ll}\text { Mass } & \text { Static } \\
\text { prop. } & \text { load }\end{array}$ \\
\hline
\end{tabular}

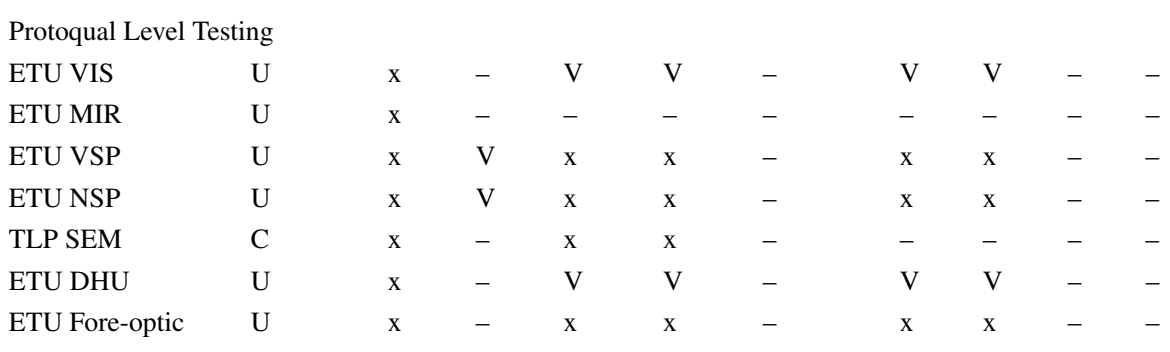

Acceptance Level Testing

\begin{tabular}{|c|c|c|c|c|c|c|c|c|c|c|}
\hline VIS & $\mathrm{U}$ & $\mathrm{x}$ & - & $\mathrm{V}$ & $\mathrm{x}$ & $\mathrm{X}$ & $\mathrm{V}$ & $\mathrm{V}$ & $\mathrm{x}$ & $\mathrm{X}$ \\
\hline NIR1/NIR2 & $\mathrm{U}$ & $\mathrm{x}$ & - & $\mathrm{X}$ & $\mathrm{X}$ & $\mathrm{X}$ & $\mathrm{X}$ & - & $\mathrm{x}$ & $\mathrm{X}$ \\
\hline NIR2-Lens & $\mathrm{C}$ & - & - & $\mathrm{X}$ & $\mathrm{X}$ & $\mathrm{X}$ & $\mathrm{X}$ & $\mathrm{x}$ & $\mathrm{X}$ & $\mathrm{X}$ \\
\hline MIR1/MIR2 & $\mathrm{U}$ & $\mathrm{x}$ & - & $\mathrm{X}$ & $\mathrm{X}$ & $\mathrm{X}$ & $\mathrm{V}$ & $\mathrm{V}$ & $\mathrm{x}$ & X \\
\hline VSP & $\mathrm{U}$ & $\mathrm{x}$ & $\mathrm{x}$ & $\mathrm{V}$ & $X$ & $\mathrm{X}$ & $\mathrm{X}$ & - & $\mathrm{x}$ & $\mathrm{X}$ \\
\hline NSP1/NSP2 & $\mathrm{U}$ & $\mathrm{x}$ & $\mathrm{x}$ & $\mathrm{V}$ & $\mathrm{X}$ & $\mathrm{X}$ & $\mathrm{X}$ & - & $\mathrm{x}$ & $\mathrm{X}$ \\
\hline TLP & $\mathrm{U}$ & $\mathrm{x}$ & - & $\mathrm{x}$ & $\mathrm{x}$ & $\mathrm{X}$ & $\mathrm{x}$ & $\mathrm{x}$ & $\mathrm{x}$ & $\mathrm{X}$ \\
\hline DHU & $\mathrm{U}$ & $\mathrm{x}$ & - & $\mathrm{X}$ & $\mathrm{V}$ & $\mathrm{X}$ & $\mathrm{V}$ & $\mathrm{V}$ & $\mathrm{x}$ & $\mathrm{X}$ \\
\hline Fiber Optic Harness & $\mathrm{U}$ & $\mathrm{x}$ & $\mathrm{x}$ & $\mathrm{X}$ & $\mathrm{X}$ & $\mathrm{X}$ & $\mathrm{X}$ & - & $\mathrm{x}$ & $\mathrm{X}$ \\
\hline POD-DHU Harness & $\mathrm{U}$ & $\mathrm{x}$ & - & $\mathrm{X}$ & $\mathrm{X}$ & $\mathrm{X}$ & $\mathrm{X}$ & - & $\mathrm{x}$ & $\mathrm{X}$ \\
\hline Heater Harness & $\mathrm{U}$ & $\mathrm{x}$ & - & $\mathrm{x}$ & $\mathrm{X}$ & $\mathrm{X}$ & $\mathrm{X}$ & - & - & - \\
\hline POD & $\mathrm{S}$ & $\mathrm{x}$ & - & $\mathrm{x}$ & $\mathrm{x}$ & $\mathrm{X}$ & $\mathrm{x}$ & - & $\mathrm{x}$ & $\mathrm{x}$ \\
\hline Instrument Deck & $\mathrm{S}$ & $\mathrm{x}$ & - & $\mathrm{x}$ & $\mathrm{x}$ & $\mathrm{X}$ & $\mathrm{X}$ & - & $\mathrm{x}$ & $\mathrm{x}$ \\
\hline Payload & $\mathrm{S}$ & $\mathrm{x}$ & - & $\mathrm{x}$ & $\mathrm{x}$ & $\mathrm{x}$ & $\mathrm{x}$ & - & $\mathrm{x}$ & $\mathrm{x}$ \\
\hline
\end{tabular}

Key: (V) Tested by instrument vendor prior to delivery to NASA Ames; (x) Tested by Ames during payload and/or spacecraft testing; (X) Test performed when unit was at a higher level of integration; (-) Not required/Not applicable/Test not performed

a Assembly Level: (C) Component, (U) Unit, (S) Subassembly

LCROSS actual powered hours, both spacecraft and payload, to requirements for spacecraft powered hours by NASA (e.g., GSFC-STD-7000 2005 more applicable to Class A missions) and DOD (e.g., MIL-STD-1540B 1982/MIL-HDBK-340A 1999, TR-2004 (8583) 2006) is shown in Table 6. After LCROSS launched, NASA Ames Research Center developed requirements for Class D Spacecraft Design and Environmental Test (APR-8070.2 2008). The LCROSS S-S/C did comply with these requirements, but the payload test program did not. Despite this, the payload performance in orbit achieved all mission requirements.

One aspect of the LCROSS payload used redundant measurements to reduce risk (see Table 2). This improved the robustness of the payload performance if a subset of instruments failed to survive through the final four minutes of the mission. The LCROSS payload design did have a potential single point failure, the DHU. However, this instrument had the most space heritage among the suite and was tested more than any of the instruments (see Table 5).

The LCROSS payload design maintained modularity to allow for easy instrument replacement during the schedule-constrained I\&T program. This was utilized three times to 
Table 5 LCROSS payload powered testing summary

\begin{tabular}{lllllll}
\hline Instrument & $\begin{array}{l}\text { Serial } \\
\text { No. }\end{array}$ & $\begin{array}{l}\text { Total hrs. } \\
\text { pre S/C- } \\
\text { integration }\end{array}$ & $\begin{array}{l}\text { Total hrs. } \\
\text { post S/C- } \\
\text { integration }\end{array}$ & $\begin{array}{l}\text { Total hrs } \\
\text { pre-launch }\end{array}$ & $\begin{array}{l}\text { Total hrs } \\
\text { pre-launch } \\
\text { (at vacuum) }\end{array}$ & $\begin{array}{l}\text { Total hours } \\
\text { on orbit }^{\mathrm{a}}\end{array}$ \\
\hline DHU & SN108 & 293.3 & 39.8 & 333.0 & 17.2 & 10.6 \\
VIS & NTSC & 131.8 & 19.4 & 151.2 & 2.3 & 4.0 \\
NIR1 & SN718 & 146.9 & 19.1 & 166.1 & 3.1 & 4.0 \\
NIR2 & SN684 & 146.6 & 20.1 & 166.6 & 3.8 & 5.1 \\
MIR1 & Thermoteknix & 135.9 & 19.1 & 155.0 & 2.3 & 4.2 \\
MIR2 & Indigo & 146.5 & 20.3 & 166.8 & 4.3 & 4.7 \\
NSP1 & SN001 & 150.0 & 19.1 & 169.1 & $5.5^{\mathrm{b}}$ & 3.7 \\
NSP2 & SN002 & 140.8 & 19.1 & 160.0 & $3.8^{\mathrm{b}}$ & 4.0 \\
VSP & ALICE-F & 146.5 & 20.1 & 166.5 & 4.1 & 4.7 \\
TLP & SEM-004 & 125.1 & N/A & 125.1 & N/A & N/A \\
TLP & SEM-003 & 0.4 & 0.6 & 1.0 & 0.0 & 0.4 \\
TLP & DEM & 125.5 & 0.6 & 126.1 & 0.0 & 0.4 \\
\hline
\end{tabular}

\section{N/A: Not-applicable}

${ }^{a}$ All hours were failure free, except TLP, which achieved $1388 \mathrm{~s}$ failure-free out of a total of $1444 \mathrm{~s}$

${ }^{\mathrm{b}}$ Failure-free hours were $1.57 \mathrm{hrs}$ and $0.48 \mathrm{hrs}$ for NSP1 and NSP2, respectively

${ }^{\mathrm{c}}$ SEM-004 failed prior to S-S/C integration. The spare SEM-003 flew, although with limited test history due to low priority level

replace three flight instruments that failed during environmental testing. The failures were related to vibration testing (NIR/Sept 2007), workmanship (TLP/Dec 2007), and vacuum testing (NSP/May 2008). For each incident, a non-conformance report was opened, the failed instrument was removed and inspected, the failure root cause was determined and documented, and the instrument was refurbished or replaced with the problem rectified. The instruments were then retested at the unit level at protoqual levels before being placed back into the assembled payload.

Payload sparing was minimal. Spares were purchased, motivated by either the criticality of the instrument (e.g., NSP and DHU) or the lead-time for ordering key components (e.g., TLP sensor or fibers). Instrument criticality was determined by the mission's science goals and requirements instrument trace (see Tables 2 and 3). The project carried full spares for the near-infrared spectrometer and visible camera (both indicated by $P$ in Table 3 ), and partial spares for long-lead time items. Instrument spares were used after launch to populate a modest payload simulator that was used for in-flight sequence development (see Sect. 5). Instrument spares were handled like flight instruments since they could have been used for flight.

The mass and power margins for the LCROSS science payload are summarized in Table 7 . The payload mass and power allocations were $40 \mathrm{~kg}$ and 100 Watts, respectively. From inspection of these tables, the payload carried large margins ( $>40 \%$ power, $>30 \%$ mass) at launch, with the exception of the bakeout heater system power. A flight rule prevented using the bakeout heaters while operating the payload instruments to avoid exceeding the power allocation. Designing and maintaining large margins within the payload subsystem, an approach used in a number of the other LCROSS spacecraft subsystems, improved overall mission risk mitigation. 
Table 6 LCROSS spacecraft and payload running times (hours) vs. standards

\begin{tabular}{|c|c|c|c|}
\hline \multirow[t]{2}{*}{ Test system or reference } & \multicolumn{3}{|c|}{ Powered time (hours) } \\
\hline & Total & Anomaly free & At vacuum \\
\hline \multicolumn{4}{|l|}{ LCROSS Program } \\
\hline LCROSS Spacecraft ${ }^{\mathrm{a}}$ & 786 & $>600$ & 412 \\
\hline LCROSS DHU & 333 & $226^{\mathrm{b}}$ & 17 \\
\hline LCROSS Science Instruments & $163^{\mathrm{c}}$ & $161^{\mathrm{d}}$ & 4 \\
\hline \multicolumn{4}{|l|}{ Test References } \\
\hline GSFC-STD-7000 (2005) (GEVS) ${ }^{\mathrm{e}}$ & 1000 & 350 & 200 \\
\hline TR-2004 (8583) (2006) & 200 & 50 & No req. ${ }^{\mathrm{g}}$ \\
\hline MIL-STD-1540B (1982)/MIL-HDBK-340A (1999) & 300 & 100 & No req. ${ }^{h}$ \\
\hline APR-8070.2 (2008) (Class D) ${ }^{\mathrm{i}}$ & 200 & 50 & 100 \\
\hline
\end{tabular}

${ }^{\mathrm{a}}$ LCROSS Project S/C Acceptance Review Package September 2008

${ }^{\mathrm{b}}$ The DHU experienced an intermittent anomaly that was tracked during the program. A software fix was put in place after which 226 anomaly-free hours were recorded

${ }^{\mathrm{c}}$ Average for all (non-DHU) instruments, except TLP. The flight TLP/SEM and TLP/DEM accumulated $1 \mathrm{hr}$ and 126 hrs burn-in, respectively, anomaly free, but only at atmospheric pressure. No vacuum testing was performed

${ }^{\mathrm{d}}$ Each NSP experienced $2.3 \mathrm{hr}$ of anomalous operation at vacuum, after which a hardware fix was needed and units re-qualified. All other instruments experienced no anomalies

${ }^{\mathrm{e}}$ General Environmental Verification Standard (GEVS) for GSFC Flight Programs and Projects

${ }^{\mathrm{f}}$ Air Force Space Command Test Requirements for Launch, Upper-Stage, and Space Vehicles

gNo requirement set for hours at vacuum, but test program must satisfy 200 hrs total burn-in

$\mathrm{h}_{\mathrm{N}}$ requirement set for hours at vacuum

${ }^{\mathrm{i}}$ Ames Procedural Requirements Class D Spacecraft Design and Environmental Test

\section{Observation Strategy}

Specific science requirements determined all LCROSS payload operations. Most important were the requirements to observe the Centaur impact at the end of the mission. All earlier payload activations supported those observations in some way, either by providing instrument calibration data or by demonstrating end-to-end system capability while in orbit that would be later needed for impact. Specific instrument operations were specified by sequences of instrument commands. These command sequences were pre-loaded to the DHU's non-volatile memory. At the time of a payload operation, a sequence start command issued from the ground in the LCROSS Mission Operations Center would start the sequence and it would proceed autonomously from there. A discrete DHU 'power-off' would power off all instruments and the DHU to end the sequence and return the payload to a survival or bakeout thermal configuration.

Table 8 summarizes the periods during which the payload was powered, their general goals, their period in the mission, and their downlink allocation. For all of these periods but the last, command sequences were pre-loaded onto the DHU. The DHU provided only 10 NVM slots, and more than 10 sequences were needed during the entire mission, so the payload was powered at other times (last line of table) to remove and load sequences. Each sequence had to meet the measurement goal requirements and fit within the allocated band- 
Table 7 Measured mass (kg) and on orbit recorded payload power (Watts) and margins

\begin{tabular}{|c|c|c|c|c|c|c|}
\hline & Power $^{\mathrm{a}}$ & Power ${ }^{b}$ & $\operatorname{Margin}(\%)^{\mathrm{b}}$ & & Power ${ }^{b}$ & $\operatorname{Margin}(\%)^{\mathrm{b}}$ \\
\hline \multicolumn{4}{|l|}{ Instrument } & \multicolumn{3}{|l|}{ Sequence } \\
\hline DHU & 13.76 & 13.76 & 86.2 & Starfield & 29.12 & 70.9 \\
\hline VIS & 3.20 & 16.32 & 83.7 & Swingby & 41.92 & 58.1 \\
\hline NIR1(2) & 2.24 & 15.36 & 84.6 & EarthCal & 41.92 & 58.1 \\
\hline $\operatorname{MIR} 1(2)$ & 1.60 & 14.72 & 85.3 & Separation & 17.60 & 82.4 \\
\hline VSP & 11.52 & 26.64 & 75.4 & Impact & 57.92 & 42.1 \\
\hline VSP (no TEC) & 4.48 & 17.60 & 82.4 & Thermal & & \\
\hline NSP1(2) & 2.88 & 16.00 & 84.0 & Survival & 58.50 & 41.5 \\
\hline TLP & 16.00 & 29.12 & 70.9 & Bakeout & 87.82 & 12.2 \\
\hline \multicolumn{3}{|l|}{ Mass } & \multicolumn{3}{|c|}{ Mass } & Margin (\%) \\
\hline \multicolumn{4}{|c|}{ Payload without panel } & \multicolumn{2}{|l|}{20.58} & - \\
\hline \multicolumn{4}{|c|}{ Panel with thermal hardware and harnesses } & \multicolumn{2}{|l|}{6.71} & - \\
\hline \multicolumn{4}{|l|}{ Total payload mass } & \multicolumn{2}{|l|}{27.29} & 31.8 \\
\hline
\end{tabular}

${ }^{\text {a }}$ Power for single instruments

${ }^{b}$ Power for DHU plus single instrument. In flight, the DHU provided either the power or directed power from the S-S/C system directly to the instruments, both requiring the DHU to be powered

Table 8 LCROSS payload operational sequences

\begin{tabular}{|c|c|c|c|}
\hline Operational sequence & Purpose & Mission period & $\begin{array}{l}\text { Data rate } \\
\text { allocation }(\mathrm{kbps})\end{array}$ \\
\hline Quick Look & Instrument health & Initial Checkout & 29 \\
\hline Star Field & Star field alignment & Pre-Swingby, Cruise & 220 \\
\hline Swingby & Calibration and alignment & Lunar Swingby & 1000 \\
\hline Moon-Earth Look & Calibration and alignment & Cruise & $29 / 60$ \\
\hline Centaur Separation & $\begin{array}{l}\text { Determination of Centaur } \\
\text { drift properties }\end{array}$ & Centaur separation & 220 \\
\hline Pre-Impact & $\begin{array}{l}\text { Instrument health, } \\
\text { calibration }\end{array}$ & $\begin{array}{l}\sim 55 \text { minutes prior to } \\
\text { Centaur impact }\end{array}$ & 1000 \\
\hline Impact-Flash & Monitor of impact flash & Centaur impact & 1000 \\
\hline Impact-Curtain & Monitor eject curtain & $\begin{array}{l}\text { From } 5 \text { sec after Centaur } \\
\text { impact to } 180 \text { seconds after } \\
\text { Centaur impact }\end{array}$ & 1000 \\
\hline Impact-Crater & $\begin{array}{l}\text { Monitor centaur impact } \\
\text { site }\end{array}$ & $\begin{array}{l}180 \mathrm{sec} \text { after Centaur impact } \\
\text { to } \mathrm{S}-\mathrm{S} / \mathrm{C} \text { impact }\end{array}$ & 1000 \\
\hline NVM Loads & $\begin{array}{l}\text { Upload new sequences to } \\
\text { DHU to be run for next } \\
\text { operation }\end{array}$ & Cruise & 29 \\
\hline
\end{tabular}


width. A more detailed description of the LCROSS mission phases can be found in Tompkins et al. (2010). The primary operational sequences are described here in more detail.

\subsection{Operational Sequences}

For each sequence, the downlink allocation was the minimum needed for the task in order to avoid using high-cost DSN assets or, in some cases, to avoid slewing the spacecraft to use the medium-gain antenna. Science data from the LCROSS mission was obtained from only the Pre-Impact and Impact (Flash, Curtain, Crater) sequences. Data from the other sequences was used for calibration and health and status.

Quick Look, a 20 minute sequence, at the lowest bandwidth, $29 \mathrm{kbps}$, powered each scientific instrument individually for about two minutes. This sequence, designed to check aliveness and health, was run many times during pre-launch I\&T as an electrical and functional baseline. In flight, it was only used once, but remained stored on board the DHU for the entire mission in case it was needed to aid in instrument troubleshooting.

During Star Field, a measurement of a specific star field was used to determine camera alignment relative to the spacecraft attitude control system. Here two cameras and the three spectrometers were powered for $\sim 60$ minutes. The actual starfield chosen was dependent on the launch date since this activity was scheduled within a few days after launch. This payload activity was the first time the $70 \mathrm{~m}$ DSN dish was used with the spacecraft's primary omni providing a test of the $220 \mathrm{kbps}$ rate. A door that covered the TLP and nadir spectrometer fore-optics during the first hours of the mission was opened during this sequence. This door protected the TLP optics and the spectrometer fore-optics in case they were pointed at the sun before attitude control was passed from the Centaur second stage to the LCROSS S-S/C. This kept the payload from imposing an attitude control requirement on the launch vehicle.

The purpose of Swingby was to make wavelength and radiometric calibration measurements and alignment measurements with all instruments (except TLP) during a lunar swingby encounter. The sequence was broken into two segments: (1) nadir viewing for calibration and inter-camera alignment, and (2) lunar limb crossings for spectrometer to camera alignment. This sequence was integrated into an absolute timing sequence on board the spacecraft to coordinate payload observations with spacecraft attitude changes. This also was the first testing of the spacecraft's medium gain antenna and the only opportunity in the baseline mission plan to test the full science rate $(1 \mathrm{Mbps})$ that would be next used in the mission's final hour 108 days later.

After swingby, during the 3.5 month cruise phase of the mission, two to three opportunities were planned to allow for observing the Earth and Moon for instrument health monitoring, contamination check, and calibration and system alignment tests. This sequence used the Quick Look sequence followed by a limb-crossing activity. These Earth-Moon Looks were also used to fine tune instrument exposure settings (see Sect. 6). In the mission, there were three Earth-Moon looks (see Table 9). The first was at optimal (near full) Earth phase at the mission's orbital minimal Earth distance. The other Earth-Moon looks were acquired within the constraints of the mission operations team and met the baseline payload calibration needs.

The Centaur Separation sequence powered two cameras to measure the dynamics of the separated Centaur. Like the swingby sequence, this sequence was integrated into an absolute timed sequence that issued the separation commands, followed by a "go-no-go" one-minute delay, which when successful, then initiated a $180^{\circ}$ degree flip of the S-S/C, activated the payload cameras for $\sim 20$ minutes and performed a braking burn to slow down the S-S/C 
Table 9 LCROSS payload on orbit activations

\begin{tabular}{lllll}
\hline Name & DOY & Date & Start UTC & End UTC $^{\text {a }}$ \\
\hline Quicklook & $09-171$ & 20-Jun-09 & $2: 41$ & $3: 03$ \\
Starfield & $09-173$ & 22-Jun-09 & $15: 50$ & $16: 50$ \\
Lunar Swingby & $09-174$ & 23-Jun-09 & $12: 18$ & $13: 06$ \\
Non-Volatile Memory Load/Dump 1 & $09-204$ & 23-Jul-09 & $15: 06$ & $16: 04$ \\
EarthCal 1 & $09-213$ & 1-Aug-09 & $21: 48$ & $22: 49$ \\
Non-Volatile Memory Load/Dump 2 & $09-227$ & 15-Aug-09 & $3: 02$ & $5: 00$ \\
EarthCal 2 & $09-229$ & 17-Aug-09 & $7: 45$ & $8: 37$ \\
Non-Volatile Memory Load/Dump 3 & $09-257$ & 14-Sep-09 & $17: 16$ & $17: 25$ \\
EarthCal 3 & $09-261$ & 18-Sep-09 & $18: 05$ & $18: 46$ \\
Separation & $09-282$ & 9-Oct-09 & $1: 51$ & $2: 09$ \\
Preimpact-Impact & $09-282$ & 9-Oct-09 & $10: 36$ & $11: 35$ \\
\hline
\end{tabular}

${ }^{\mathrm{a}}$ The UTC times have been rounded to the nearest minute

to follow the Centaur by a four minute separation. The separation event occurred approximately 9 hours, 40 minutes prior to Centaur impact, when the $S-S / C$ was $\sim 40,000 \mathrm{~km}$ from the Moon.

The first $\sim 55$ minutes of the final hour of descent was spent in the Pre-Impact sequence to check instrument health, provide contextual descent data and instrument calibrations. Here, a cadence of $0.82,0.41,0.41,3.0$, and $3.0 \mathrm{~Hz}$ was specified for the VIS, NIR1, NIR2, MIR1, and MIR2 cameras, respectively. VSP was specified to operate in bracket mode, a triplet of 500, 100 and $2500 \mathrm{~ms}$ spectra at $0.2 \mathrm{~Hz}$ cadence. NSP1 and NSP2 each operated in their $1.7 \mathrm{~Hz}$ Hadamard mode. The TLP was not powered.

About 58 seconds prior to the Centaur impact, the Impact sequence started. This second sequence had three sub-sequences each optimized for specific elements of the final four minutes: Flash, Curtain, and Crater. The Impact sequence also powered the TLP (the only time this instrument was used in the mission) at 24 minutes prior to the Centaur's impact.

In Flash, the instruments were configured to optimize measurements of the Centaur's impact flash. The key instruments were NIR1, TLP and the spectrometers. The goals of this period were to identify the location of the flash, capture the flash in the visible and near infrared, make a time resolved measurement of the total power of the flash and measure the visible and near-infrared spectrum of the flash. Data from the TLP and NSP1 (in Flash mode) at $1 \mathrm{kHz}$ and $72 \mathrm{~Hz}$, respectively, affected the total bandwidth, so not all instruments could stream data in this one-minute period. VIS and NIR2 were disabled. NIR1, MIR1 and MIR2 were specified to operate at 3.0, 0.5 and $0.5 \mathrm{~Hz}$, respectively. The VSP stayed in bracket mode, characterized by three 2 -second exposures (with a gap of $<7 \mathrm{~ms}$ in between), one of which was expected to capture the visible flash. In the mission design, uncertainties in the impact time, command time and S-S/C clock time each at most had an error of \pm 1 second.

In Curtain, the instruments were configured to optimize measurements of the evolution of the solar-illuminated ejecta curtain. The goals of this $\sim 3$ minute period were to monitor the ejecta curtain, measure the curtain evolution to estimate the total ejecta mass, monitor the ejecta curtain's thermal evolution and obtain infrared image pairs. The key instruments were VIS, both NIR1 \& NIR2, and the two nadir spectrometers (VSP \& NSP1). The cadence 
of the instruments was identical to that used in Pre-Impact. The TLP was powered off at the beginning of this sequence.

In Crater, the instruments were configured to optimize measurements of the crater formed by the Centaur impact itself. This sequence occurred in the last minute of the mission. The goals of this sequence were to image the Centaur impact crater, improve identification of the impact crater's location and monitor the ejecta cloud with the side-viewing spectrometer. The key measurement instruments were the two mid-infrared cameras (MIR1 \& MIR2) and NSP2. To maximize MIR coverage temporally, $3 \mathrm{~Hz}$ per camera was specified. VIS and NIR1 were disabled in this period. NSP1 and NSP2 remained in Hadamard mode $(72 \mathrm{~Hz})$ and the VSP was configured to operate at a $0.52 \mathrm{~Hz}$ cadence, the fastest the DHU could reliably drive the VSP for a bracket mode of 200, 100, and $400 \mathrm{~ms}$. The remaining bandwidth allowed NIR2 at $0.67 \mathrm{~Hz}$. During this sequence, the S-S/C was expected to potentially fly through the debris cloud through which the solar near infrared spectrometer (NSP2) measurements in those final few seconds could provide additional measurements of the ejecta curtain.

In practice, the actual executed Pre-Impact and Impact sequences deviated from the plan due to bandwidth limitations and scene complexity. This is described in more detail in Sect. 6.6.

The total mission downlink volume for the LCROSS science instruments was 909,198,000 bytes $(900 \mathrm{Mb})$. The size of the raw and calibrated products, trajectory information (e.g., SPICE kernels) and documentation to the Planetary Data System (PDS) was 10 Gbytes. Note that the LCROSS payload was powered off for the majority of the 112 LCROSS mission. The unpowered payload was kept in bakeout mode at a modest $+35-$ $40^{\circ} \mathrm{C}$ temperature for the majority of the mission to reduce contaminants. This was possible because the S-S/C maintained large power margins throughout the mission.

\subsection{Payload Simulator}

Spacecraft simulators are used for a variety of purposes including operator training, anomaly resolution, and flight software and command product development and testing. LCROSS was no exception. The LCROSS spacecraft simulator was sufficiently complete and accurate to support these uses, but, for budgetary reasons, the payload portion of the simulator was more limited. It was missing copies of the TLP, NIR2, MIR2 and NSP2, and was not integrated electrically into the spacecraft simulator. A manual switch powered the payload simulator rather than software command sourced from the spacecraft simulator's power subsystem.

The short lifetime and uniqueness of the DHU (i.e., a novel build for LCROSS despite being made of high heritage Ecliptic DVS components) prohibited the development of a typical software/mock-hardware rack-mount simulator. The solution was the purchase of a second DHU unit that also served as the flight spare DHU. This spare DHU also became essential for the development and testing of new sequences that had to be modified from pre-launch baselines.

Baseline instrument command sequences were developed before launch and validated on the flight payload. The limitations of the payload simulator meant there was risk involved in changing these sequences during flight. Only limited changes were allowed to later sequences based on what had been learned from executing earlier ones. The simulator's weakest area was in scene generation, that is, the ability to mimic the scene that would be observed by the payload suite at various times during the mission. This was improved by purchasing a VGA to NTSC convertor that allowed for synthesizing visible and near 
infrared camera images. This synthesis method bypassed the cameras themselves but did test DHU image capture, compression and downlink. This approach was added late in the mission (post launch) and did not cover the thermal cameras. As a result, in practice it was discovered that it had not adequately matched the actual lunar scenes, causing significant problems and the need for using in flight operation mitigations (see Sect. 6).

\section{In-flight Performance and Calibration}

The following paragraphs summarize key observations of the payload performance and the calibration checks performed in flight. The date and duration of all flight instrument operations are summarized in Table 9. Within the 112-day mission, the DHU was powered a total of 13 times. The other science instruments were powered $\sim 5-8$ times, as needed by the sequences.

All instruments demonstrated exceptional performance on orbit, with one exception. The TLP, whose original SEM failed before spacecraft integration (see Table 5), had a replacement SEM that potentially contained the workmanship error found in the failed unit after a root cause was determined. By this time in the payload I\&T schedule, an operational workaround (i.e., power it once in flight) was chosen over replacing the SEM. Therefore, the TLP was powered once during the mission at $\sim 24$ minutes prior to Centaur impact sequence. In operations, careful monitoring was made of this instrument to look for deviations from prior test baselines. None were detected. At 09-282 11:33:20 UTC, the TLP showed behavior in line with the failure from the previous SEM, but this occurred 150 seconds after the Centaur impact, when the TLP's usage was no longer needed. The on board sequence eventually terminated the TLP power to direct the bandwidth margin towards the thermal cameras in the final minute. Therefore, the TLP performed per design for 1388 seconds out a total of 1444 seconds, or $96 \%$ of it allotted time, with $100 \%$ performance covering the time it was designed to cover scientifically. Although the TLP did not observe the visible flash, it did observe changes in the curtain. The flash's near-infrared peak and decay was detected in the NSP1. The VSP did detect the flash, but the instrument had been set for a two second integration period so a single spectrum contained the flash's visible component plus the beginnings of the curtain expansion. The absence of the flash detection by the TLP provided information addressing the material type impacted by the Centaur (Schultz et al. 2010).

Prior to launch, all instruments underwent calibration to verify that the instruments would meet their measurement requirements. Specifically, wavelength and radiometric calibration for the three spectrometers and photometer, and dynamic range calibration of the near and mid-infrared cameras was performed. Radiometric calibration for the visible camera was prevented as it had been set by the vendor to an automatic mode without an ability to query its gain or exposure settings. The LCROSS payload did not carry on-board calibration sources, an approach typically used for longer duration missions. This meant that the calibration data products (e.g., for the PDS) were based on pre-launch calibration data. Therefore, any pre-launch absolute radiometric calibration testing was performed at a level to provide sufficient guidance for exposure settings and saturation checks.

\subsection{Cosmic Rays}

Effects of cosmic rays were observed in the cameras and spectrometers on the LCROSS payload. No significant permanent damage was observed, nor did any of the instruments or 

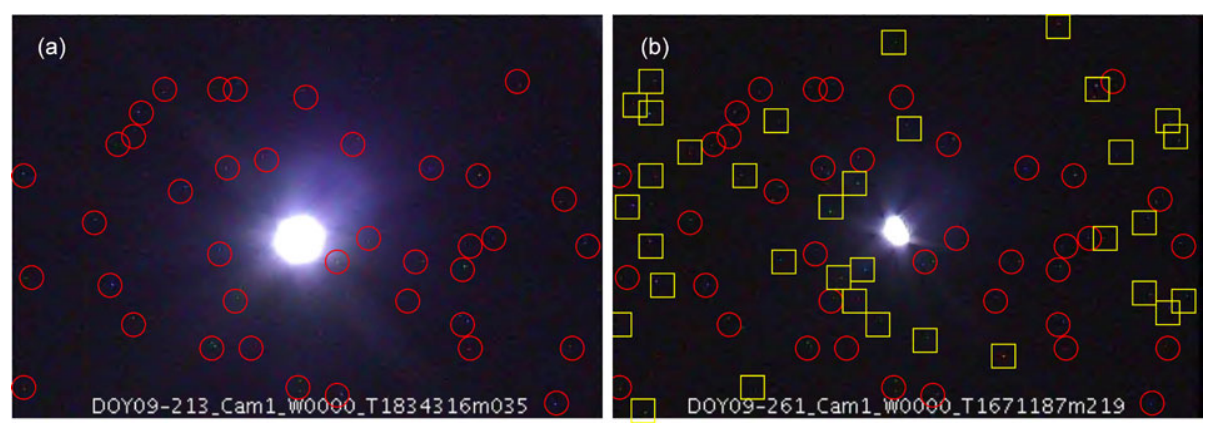

Fig. 4 VIS images taken during Earth observations on mission day (a) DOY09-213, day 44, and (b) DOY09-261, day 93. In both images, the VIS full $30.1^{\circ} \times 22.8^{\circ} \mathrm{FOV}$ is shown. Differences in the size of the Earth were due to the different phases and distance from S-S/C ((a) $157^{\circ}$ phase, $360,000 \mathrm{~km},(\mathbf{b}) 105^{\circ}$ phase, 560,000 km). 'Hot pixels' observed on day 44 are circled in red and remained high in the data on day 92, shown also as red circles. The yellow squares show new 'hot pixels' that were not present on day 44 but were present in the day 92 data. The VIS flight configuration set the exposure to auto-exposure. It overexposed on the Earth because its on-board algorithm optimized on the dark sky background that dominated the scene area. Although the majority of the VIS images during the LCROSS mission contained the bright moon for which the on-board exposure time was decreased and hot pixels were not as noticeable, inspection of VIS images taken when the camera stared at dark sky and was expected to have to longer integrations, supports the trend of permanently damaged pixels for this COTS instrument. The actual exposure times used in any VIS images were not transmitted in the telemetry

the DHU suffer any single event upsets due to cosmic rays. The visible camera demonstrated some permanent hot pixel damage (Fig. 4). The near infrared camera also was susceptible (see Sect. 6.2), but subsequent images of similar scenes showed that this array recovered with no permanent damage. For the 112-day mission, the payload was powered at most $10.6 \mathrm{hrs}$, or $0.4 \%$ of the mission lifetime. It should be noted that no specific additional radiation shielding was added to these COTS instruments, a decision based on the known short mission lifetime and short powered operation scenarios.

During the first four days of the mission, en route to the moon, the visible camera, NIR cameras and the UV-visible spectrometer showed significant numbers of pixels affected by cosmic rays. In particular, using the UV-visible spectrometer as a guide, LCROSS observed a cosmic ray count gradient change qualitatively similar to that observed by LRO's CRaTER instrument on its journey to the moon during the same time period. CRaTER's data showed how the rates dropped as LRO approached the Moon owing to a geometric effect (Spence et al. 2010). Unlike CRaTER, the LCROSS UV-visible spectrometer could not distinguish different energies or even distinguish multiple vs. single source, due to the co-adding within the instrument before data transfer to ground.

\subsection{Alignment Verification and Geometric Calibration}

The UV-visible spectrometer (VSP) and nadir-looking near infrared spectrometer (NSP1) each had the smallest FOVs among the LCROSS instruments: a circular $1^{\circ}$ diameter FOV. Their fore-optics' optical prescription was a non-focused system designed to optimize throughput, acting as a "light bucket." Any light within this $1^{\circ}$ diameter FOV would be detected and dispersed within the spectrometer.

The center of the nadir near-infrared spectrometer (NSP1)'s $1^{\circ}$ diameter FOV was defined as the payload boresight, to which all instruments centers/edges were measured. In the final hour of the LCROSS mission, the S-S/C was maneuvered to point this boresight at the 

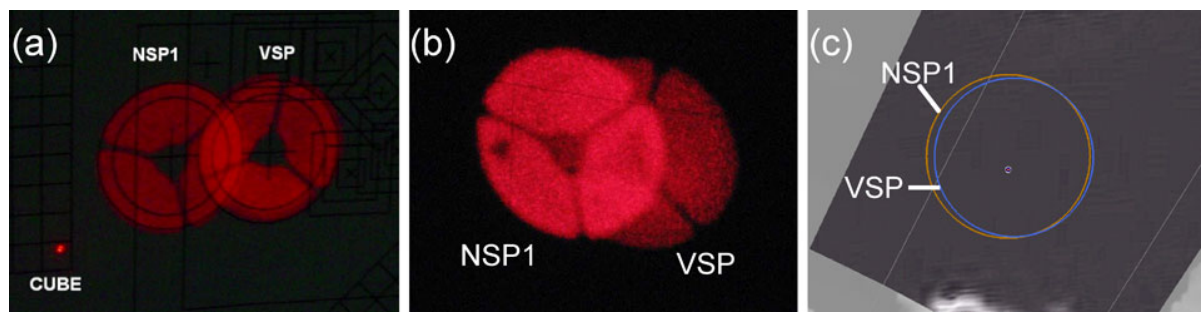

Fig. 5 Knowledge of the co-alignment and FOV of the nadir spectrometers was obtained using a series of tests where the fore-optics' centers, projected FOV sizes, and offsets were measured over a range of distances and orientations. VSP and NSP1 have FOVs of $0.98 \pm 0.01^{\circ}$ and $1.01 \pm 0.04^{\circ}$, respectively, and aligned to within $0.1^{\circ}$. The vectors representing the centers of these fore-optics, taking into account measured angular offsets, were calculated. The NSP1 vector (in the spacecraft body frame) defined the payload boresight. (a) Photo of a test target in the lab that was used to tie all nadir instrument centers and FOVs to the NSP1 center. The VSP and NSP1 fore-optics have been back-illuminated by a laser. This photo also shows a reflected laser light from the POD cube that was used to tie the POD cube orientation to the NSP1 center The NSP1 and VSP are not overlapping here because the test target was too close to the POD entrance aperture. The POD cube also provided the transformation to spacecraft body frame coordinates when measured with other cubes placed on the S-S/C. (b) Photo of the back-illuminated NSP1 and VSP fore-optics at $8.5 \mathrm{~m}$ distance during FOV and co-alignment monitoring through environmental testing. (c) The prediction for the NSP1 and VSP FOV overlap at $599 \mathrm{~km}$ (the distance between the S-S/C and the Moon at the Centaur impact) superimposed on a NIR2 image. The dot in the center is the estimated target location (see Fig. 9)

calculated Centaur impact location. To meet the requirement that the NSP1 and VSP observe the same scene of the evolving ejecta curtain, these instruments, each with their $1^{\circ}$ diameter FOV, had to be co-aligned to within $0.1^{\circ}$. Prior to launch, both the FOVs and their coalignment were repeatedly measured the lab and verified they did not change as a result of vibration and thermal environmental changes. To measure the FOVs and their co-alignment, the VSP and NSP1 fore-optics were back-illuminated with a collimated beam from a laser, a technique suggested by the vendor (Fig. 5). For the formal verification testing, the throw distance of 8.5 meters was sufficient to provide a measurement of a $0.1^{\circ}$ co-alignment within the set-up errors. The VSP and NSP1 FOVs were measured to be $0.98 \pm 0.01^{\circ}$ and $1.01 \pm$ $0.04^{\circ}$, respectively.

To tie the intra-instrument alignment to the S-S/C coordinate system, an alignment cube was placed on the POD, and the angles between the NSP1's FOV center and the cube were measured using a combination of direct lasers and theolodites. At the spacecraft level, the POD cube's position was measured and tracked (through environmental testing) with respect to other cubes on the spacecraft. Prior to launch, these tests performed by NASA Ames and NGST using alignment cubes defined the LCROSS payload pre-launch boresight as $\{X, Y, Z\}=\{0.999879,0.00794955,0.0133901\}$ in the spacecraft body frame coordinates. The boresights of each payload instrument in spacecraft body frame coordinates are defined in Table 10.

To provide an early gross $\left(<5^{\circ}\right)$ check for shift in payload alignment due to launch, the $\mathrm{S}-\mathrm{S} / \mathrm{C}$ was commanded to point the payload boresight at a star field. For the June 18, 2009, launch date, this star field centered on Altair. A finer $\left(<0.1^{\circ}\right)$ alignment measurement would be accomplished using a lunar limb scan during swingby later in the mission (see below).

During the Star Field observation, the unfiltered near infrared camera (NIR2) was set at its longest exposure time, OPR15, $16.24 \mathrm{~ms}$. NIR2 was operated without and with enhancement, the latter where pixel data was linearly stretched over the available pixel bit depth to result in a higher contrast image. The images provided several stars for alignment verification. The three brightest are called out in Fig. 6. Hot pixels, which re- 
Table 10 LCROSS instrument center boresight vectors in spacecraft body frame coordinates

\begin{tabular}{llrr}
\hline & \multicolumn{1}{l}{$Y$} & \multicolumn{1}{l}{$Z$} \\
\hline NSP1 & 0.999879 & 0.007950 & 0.013390 \\
NSP2 & 0.001753 & -0.141368 & -0.994075 \\
VSP & 0.999877 & 0.008528 & 0.013176 \\
VIS & 0.999840 & 0.017651 & 0.002948 \\
NIR1 & 0.999724 & 0.021583 & -0.009296 \\
NIR2 & 0.999645 & 0.025364 & -0.008136 \\
MIR1 & 0.999975 & -0.001297 & -0.006999 \\
MIR2 & 0.999957 & 0.008967 & 0.002387 \\
TLP & 0.999879 & 0.007950 & 0.013390 \\
\hline
\end{tabular}
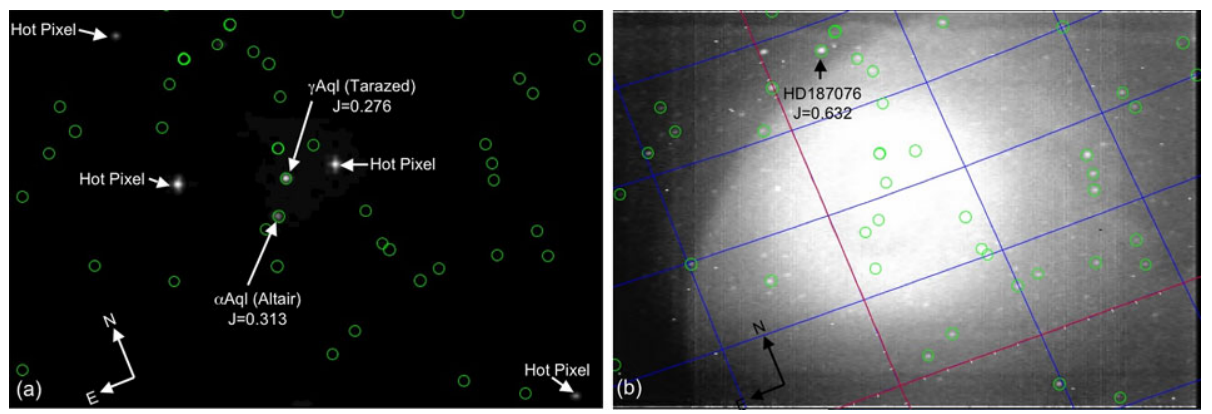

Fig. 6 The LCROSS near-infrared camera (NIR2) observing a star field. (a) A single image taken at the camera's longest exposure time $(16.24 \mathrm{~ms})$. Three bright stars $(J<0.6)$ are easily detected. (b) A single image taken with the camera in enhanced-mode at the same exposure time and in the same orientation as in (a). A RA/Dec grid is overlaid. In enhanced-mode, the camera's pixel data was linearly stretched over the available pixel bit depth to result in a higher contrast scene for display. Enhanced mode at 16.24 ms revealed stars $(J<0.9)$ near the edges in a single image. The central bright area on this image was a flat field artifact of this mode, a mode used only once during the star field observation. The green circles are from a WCS fit to twenty-seven 2MASS stars (RMS residuals to the fit were 250 arc-seconds or 1.3 pixels). Pixel scales in the two orthogonal directions are 149.3 and 164.7 arcsec/pixel. Rotation with respect to equatorial north is 21.8 degrees

mained high for the entire $\sim 50$ minute sequence are also identified. Those pixels did not move with time as the other stars did due to the motion of the attitude control system deadband. In the left image (non-enhancement), two stars dominate near the image center. These were expected to be $\alpha$ Aquila (Altair) and $\gamma$ Aquila (Tarazed), which have an angular separation of $\sim 2$ degrees. The NIR2 bandpass is $0.9-1.7 \mu \mathrm{m}$, covering most of the I band and the entire $\mathbf{J}$ band. Inspection of I, J \& $\mathrm{H}$ colors indicated that Tarazed should be brighter than Altair for the NIR2 response function. The right image (enhancement on) provided confirmation of the correct identification of $\alpha$ Aquila (Altair) and $\gamma$ Aquila (Tarazed) using fainter stars and confirmed the brighter star is Tarazed. Final identification was performed by overlaying a world-coordinate-system (WCS) fit of the 2MASS catalog (green circles) to the image data (Greisen and Calabretta 2002; Skrutskie et al. 2006).

The measured location of $\gamma$ Aquila and $\alpha$ Aquila (the target) indicated an offset of 1.63 and 0.47 degrees, respectively from the NSP1 boresight indicating no large misalignments due to launch. The camera platescale of $0.4^{\circ} /$ pixel was also confirmed by these images. The 
Fig. 7 LCROSS visible camera image mosaic during lunar swingby (DOY09-174) with marked locations of the three ground target centers and the two alignment limb crossings.

Target 1 (Mendeleev) was chosen as being old highland material. The large size of Mendeleev $(\sim 313 \mathrm{~km})$ provided a fairly uniform target. At Target 1, LCROSS was at $\sim 8200 \mathrm{~km}$ altitude: the NSP1/VPS FOVs subtended a $143 \mathrm{~km}$ diameter circle. Target 2's (Goddard C) crater floor had been resurfaced in the past by lava floods, leaving the interior nearly flat and featureless. Goddard C itself is $140 \mathrm{~km}$ in diameter. At Target 2, LCROSS was at $\sim 8800 \mathrm{~km}$ altitude: the NSP1/VPS FOVs subtended a $154 \mathrm{~km}$ diameter circle. Target 2 also aligned with the termination on DOY09-174. Target 3 (Giordano Bruno) is at the center of a symmetrical ray system of ejecta extending out $150 \mathrm{~km}$ in diameter that has a higher albedo than the surrounding surface. At Target 3, LCROSS was at $\sim 9300 \mathrm{~km}$ : the NSP1/VPS FOVs subtended a $164 \mathrm{~km}$ diameter circle. The two limb crossings are orthogonal to each other

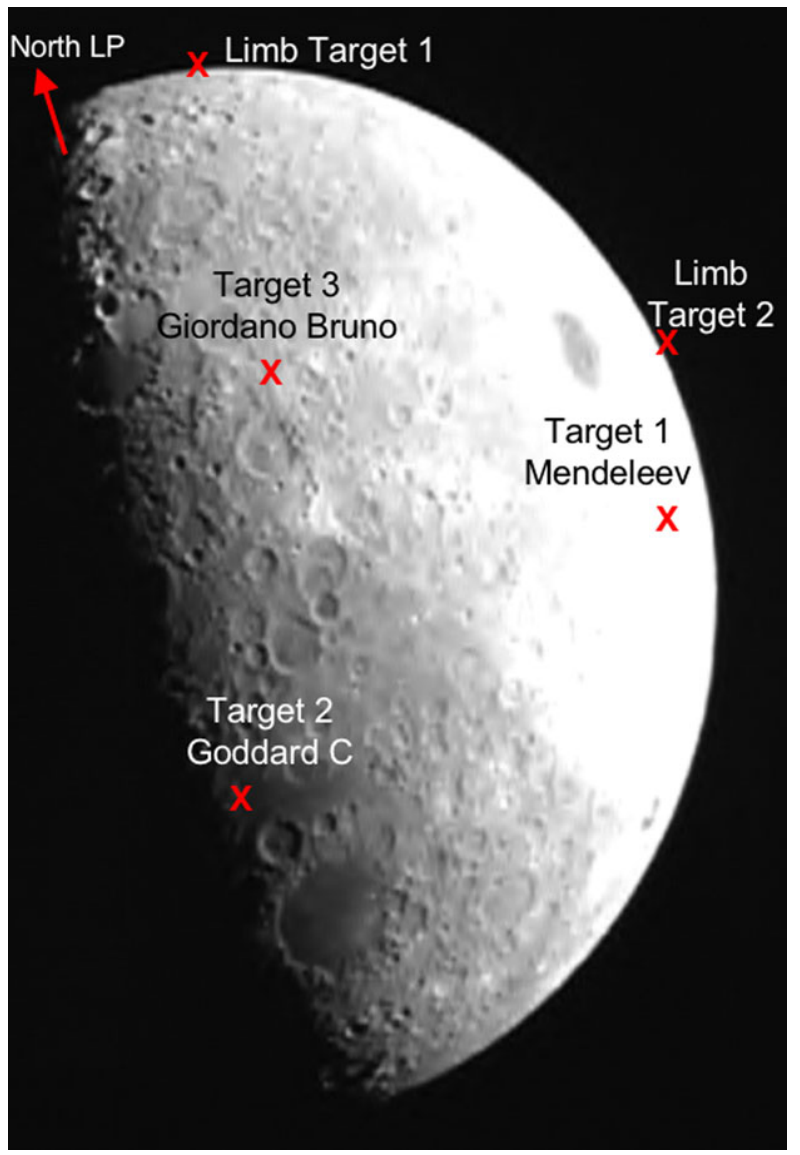

near infrared camera, operating in 8-bit, with a low infrared throughput lens, was able to detect stars down to $J=0.6 \mathrm{mag}$.

Since the star field result indicated the payload alignment had not significantly shifted due to launch, the mission designers used the pre-launch boresight for design of the swingby $\mathrm{S}-\mathrm{S} / \mathrm{C}$ pointing sequence. The three ground targets and two limb locations are shown in Fig. 7. Seven limb crossings were observed by both nadir spectrometers (VSP \& NSP1) (Fig. 8). The spacecraft swept the instruments across the lunar limb at two points, roughly 90 degrees apart. LCROSS slewed at $0.2^{\circ} / \mathrm{s}$, during which spectra from the nadir spectrometers were taken continuously at $0.5 \mathrm{~Hz}$ and $72 \mathrm{~Hz}$, for VSP and NSP1, respectively. The spectrometer boresights were estimated by normalizing the actual signal and comparing it against a predicted signal generated by: (1) estimating a boresight vector (and therefore FOV) relative to the Star Tracker; and (2) computing the percentage of the FOV filled by moon vs. empty space by computing the geometric intersection of the moon's disk with the FOV. An algorithm searched through possible boresight vectors, minimizing the root-mean-square error between the predicted and actual signals. The largest source of error in this approach was time synchronization between the ground, spacecraft and instruments. The NSP1 post-launch measured boresight in spacecraft body frame coordinates is $\{X, Y, Z\}=\{0.99987989,0.00680375,0.01392503\}$. This was $0.06 \pm 0.02$ delta-degrees from pre-launch boresight (see above). Subsequent Earth calibration crossings, when the 


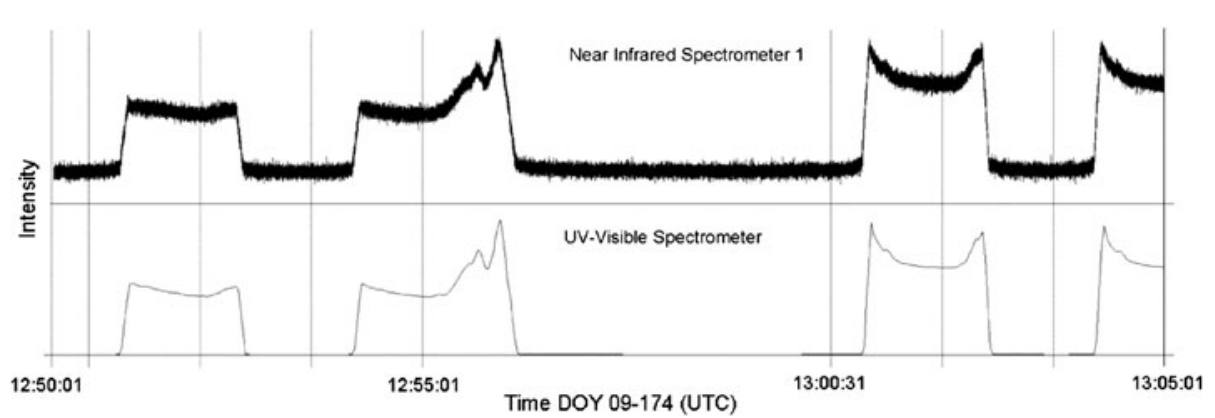

Fig. 8 Time trace of integrated (top) NSP1 and (bottom) VSP spectra during lunar swingby limb crossings. The limb observations were measured over 15 minutes. All seven crossings were utilized to calculate the payload boresight

Earth underfilled the spectrometer FOVs, provided additional gross checks, but were at a lower fidelity than the lunar limb data. Because the calculated on-orbit boresight measurement derived from swingby met the pointing requirements $\left(<0.1^{\circ}\right)$, the pre-launch boresight was kept for the remainder of the mission.

The FOVs and orientations of all eight nadir instruments is summarized in Fig. 9a. The payload boresight is the center of the NSP1 FOV. The backdrop is a generated lunar surface with lighting conditions to match that at 2009-10-09 11:31:19.506 UTC, the time of the Centaur impact. Satellite Toolkit (STK) ${ }^{1}$ planning tools were used to propagate the trajectory vectors to overlay the centers, extents and angles of the FOVs of the instruments. The overlapping boresights of the NSP1 and VSP are shown in greater detail in Fig. 9b. The target spot in the center of Fig. 19b is an estimate of the impact location (Marshall et al. 2011). This analysis shows that NSP1's FOV did encircle the impact point, a measurement also substantiated with the flash detection by the NSP1 (Schultz et al. 2010). The impact point was also correlated with the ejecta cloud detected a few seconds later in the VIS camera.

Two cameras were not at optimal focus during the mission, NIR1 and MIR2. NIR1 was a known issue prior to launch. MIR2, however, defocused post-launch. This was first noticed during lunar swingby. There was one opportunity during the mission to put a bright point source on MIR2, using the Moon during the second Earth-Moon Look, on DOY09-229, to provide data to reconstruct the point-spread-function (PSF). PSFs were not measured for any of the cameras prior to launch. In this observation, the S-S/C was $\sim 520,000 \mathrm{~km}$ and $\sim 881,000 \mathrm{~km}$ from the Earth and Moon, respectively. At these distances, the Earth and Moon subtended $\sim 1.6^{\circ}$ and $0.15^{\circ}$, respectively. This meant, the Moon subtended a single MIR pixel (see Sect. 3.3, iFOV $=0.097^{\circ} \times 0.097^{\circ}$ ). This was confirmed in the focused MIR1 images. Geometric corrections for the cameras, either correcting for non-square pixels or focus issues, were not part of the delivery to the PDS. Users of the LCROSS camera data should take into account the VIS and NIR non-square pixels during analysis. The curtain measurements derived from the VIS camera did take into account the rectangular pixels (Schultz et al. 2010). The Star Field mapping measurements discussed above (see Fig. 6) also corrected for non-square pixels. Series of pre-impact images have been shown to provide geometric corrections for the cameras that may be useful for users of LCROSS camera data (see Moratto et al. 2010).

\footnotetext{
${ }^{1}$ Satellite Toolkit, Analytical Graphics Inc., 220 Valley Creek Blvd., Exton, PA 19341, USA, Phone: 610981-8888, E-mail: info@agi.com,www.agi.com.
} 
Fig. 9 The FOVs of the nadir-looking instruments are shown superimposed against a STK-generated backdrop with the lighting conditions at the time of impact, 2009-10-09

11:31:19.506 UTC. In this view, the $\mathrm{S}-\mathrm{S} / \mathrm{C}$ is $599 \mathrm{~km}$ to the impact point. (a) The FOVs and relative orientations. VIS $\left(30.1^{\circ} \times 22.8^{\circ}\right)$, NIR1 $\left(28.7^{\circ} \times 21.7^{\circ}\right)$ and MIR1 $\left(15^{\circ} \times 11^{\circ}\right)$ are shown with solid boxes with colors light-blue, orange, and red, respectively. NIR2 $\left(28.7^{\circ} \times 21.7^{\circ}\right)$ and MIR2 $\left(15^{\circ} \times 11^{\circ}\right)$ are dashed boxes with colors orange and red, respectively. The $10^{\circ}$ dia. TLP FOV is shown by the purple circle. In this view, the $1^{\circ}$ dia. NSP1 and VSP FOVs overlap, orange and blue circles, respectively. (b) The NSP1 and VSP $1^{\circ} \mathrm{FOV}$ s at this same altitude, but the view has been magnified to just show these smallest FOVs with respect to the surroundings. These

surroundings are represented here by a portion of an NIR2 image taken at the time of impact. This area differs from the larger background, which was synthesized from an LRO/LOLA terrain dataset and a lighting model. An estimate of the impact location is indicated by the labeled dot (Marshall et al. 2011)
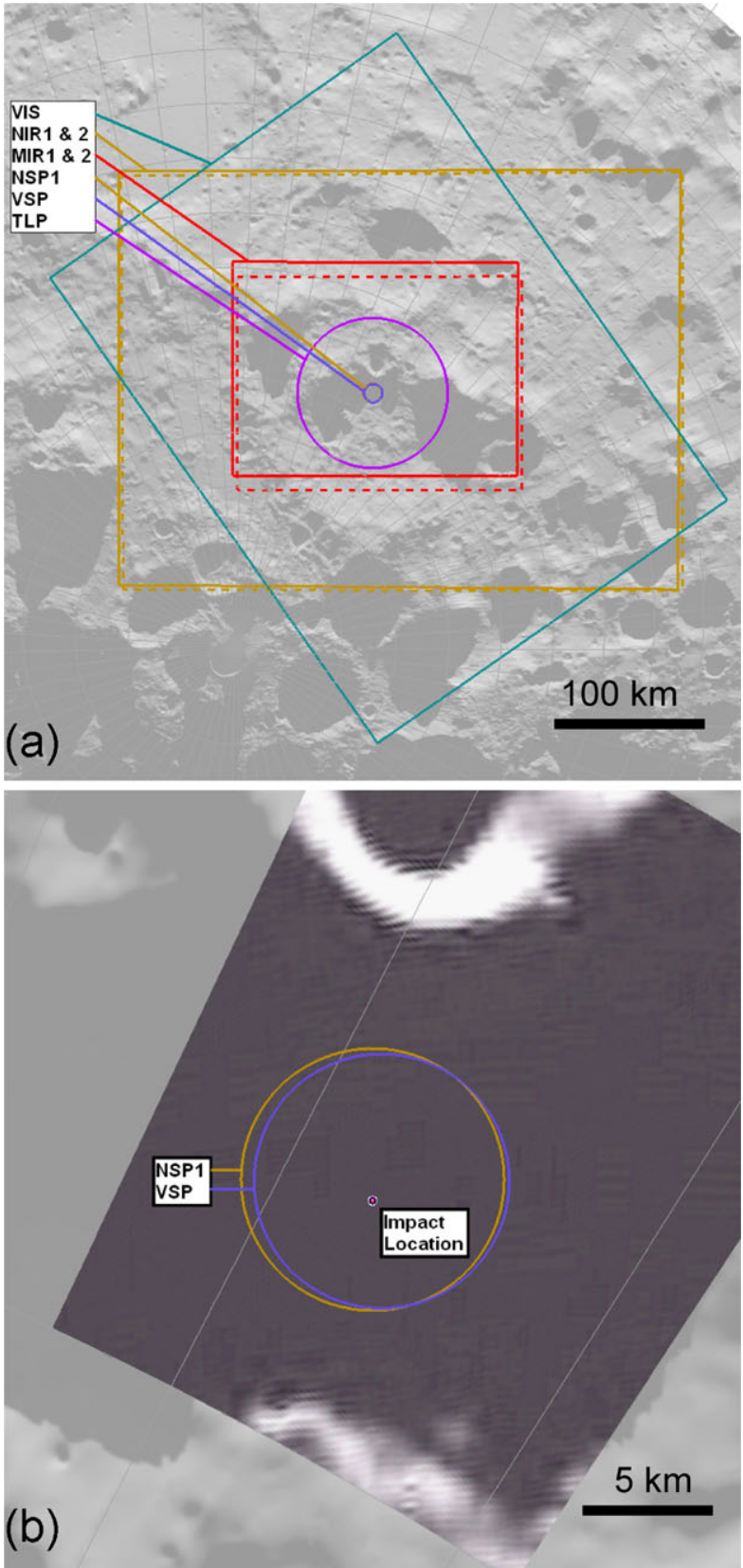

\subsection{Spectrometer Calibration}

\subsubsection{UV-visible Spectrometer}

In its design, the VSP's optical prescription created a two-dimensional spectrum onto a single $1044 \times 64$ pixel Hamamatsu CCD detector. The spectrometer then internally row 


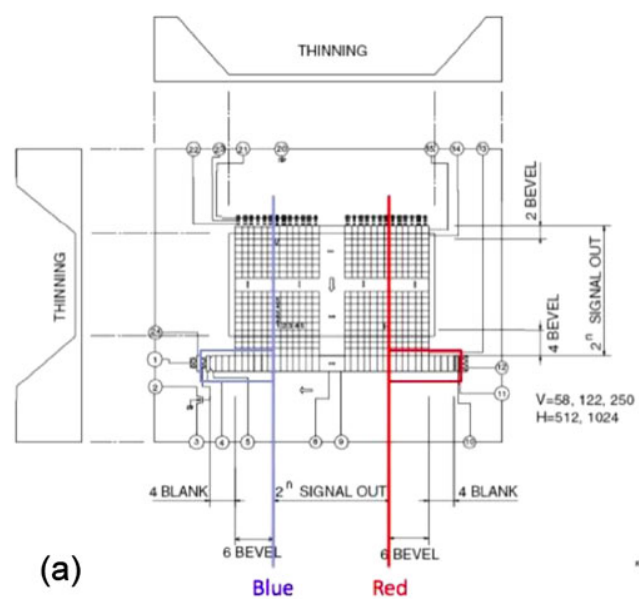

\begin{tabular}{|c|c|}
\hline Pixel & Description \\
\hline 0 & Blue Bevel \\
\hline 1 & Spectral Pixel 0 \\
\hline 2 & Spectral Pixel 1 \\
\hline$\ldots$ & $\ldots$.Spectral Pixels... \\
\hline 1023 & Spectral Pixel 1022 \\
\hline 1024 & Spectral Pixel 1023 \\
\hline $1025-1030$ & Red Bevel \\
\hline $1031-1034$ & Red Dark/Blank \\
\hline $1035-1037$ & Blue Dark/Blank \\
\hline
\end{tabular}

Fig. 10 (a) The 1044 column $\times 64$ row pixel Hamamatsu CCD detector layout used in the LCROSS VSP. 64 rows are co-added before downlink, providing a 1044 pixel 1-D spectrum per acquisition. The 1044 pixels contain 1024 spectral pixels and 20 housekeeping $(\mathrm{H} / \mathrm{K})$ pixels. These $\mathrm{H} / \mathrm{K}$ pixels have two varieties, blank/dark (i.e. covered to block light) and bevel (i.e., sensitive to light but outside the optical path). (b) The actual mapping of the spectral, blank (dark), and bevel pixels for the flight VSP data (in the PDS) is summarized here. The blue/red designations reflect the physical location on the CCD. There is a known software feature onboard the VSP that created the odd numbering shown in (b)

Table 11 Gaussian-fitted resolved arc line profiles used for VSP resolution measurements

\begin{tabular}{llllll}
\hline $\begin{array}{l}\text { Wavelength } \\
(\mathrm{nm})\end{array}$ & $\begin{array}{l}\text { Arc line } \\
\text { FWHM } \\
(\mathrm{nm})\end{array}$ & Resolution & $\begin{array}{l}\text { Wavelength } \\
(\mathrm{nm})\end{array}$ & $\begin{array}{l}\text { Arc line } \\
\text { FWHM } \\
(\mathrm{nm})\end{array}$ & Resolution \\
\hline 289.01 & 1.01 & 286 & 435.65 & 0.73 & 598 \\
296.38 & 1.01 & 292 & 507.14 & 0.67 & 760 \\
301.84 & 1.06 & 286 & 545.90 & 0.63 & 862 \\
312.60 & 1.30 & 240 & 576.80 & 0.68 & 845 \\
364.82 & 1.14 & 321 & 621.52 & 0.73 & 847 \\
404.45 & 0.78 & 516 & 630.29 & 0.72 & 873 \\
\hline
\end{tabular}

co-added the pixels to deliver a 16-bit, $1 \times 1044$ pixel spectrum to the LCROSS DHU electronics for downlink. The pixels contained both spectral and housekeeping dark reference data, the latter was used for a dark subtraction step in the radiometric calibration (see below). The physically layout and description of these spectral and housekeeping pixels are shown in Fig. 10.

Pre-launch lab-based wavelength and resolution calibration for the UV-visible spectrometer was provided using a series of Neon and HgAr arc lamps. The VSP's spectral resolution ( $R \sim 300-850$ over its wavelength range) was measured by fitting Gaussian profiles to $\mathrm{HgAr}$ and He arc lamps during pre-launch testing. Resolved line FWHM values across the VSP wavelength range is summarized in Table 11.

On-orbit wavelength calibration was performed using high signal-to-noise reflectance spectra from the lunar surface obtained during lunar swing-by on DOY09-174. Over 150 strong solar lines dominated the spectrum (Fig. 11). Fifty-one of the strongest absorption 


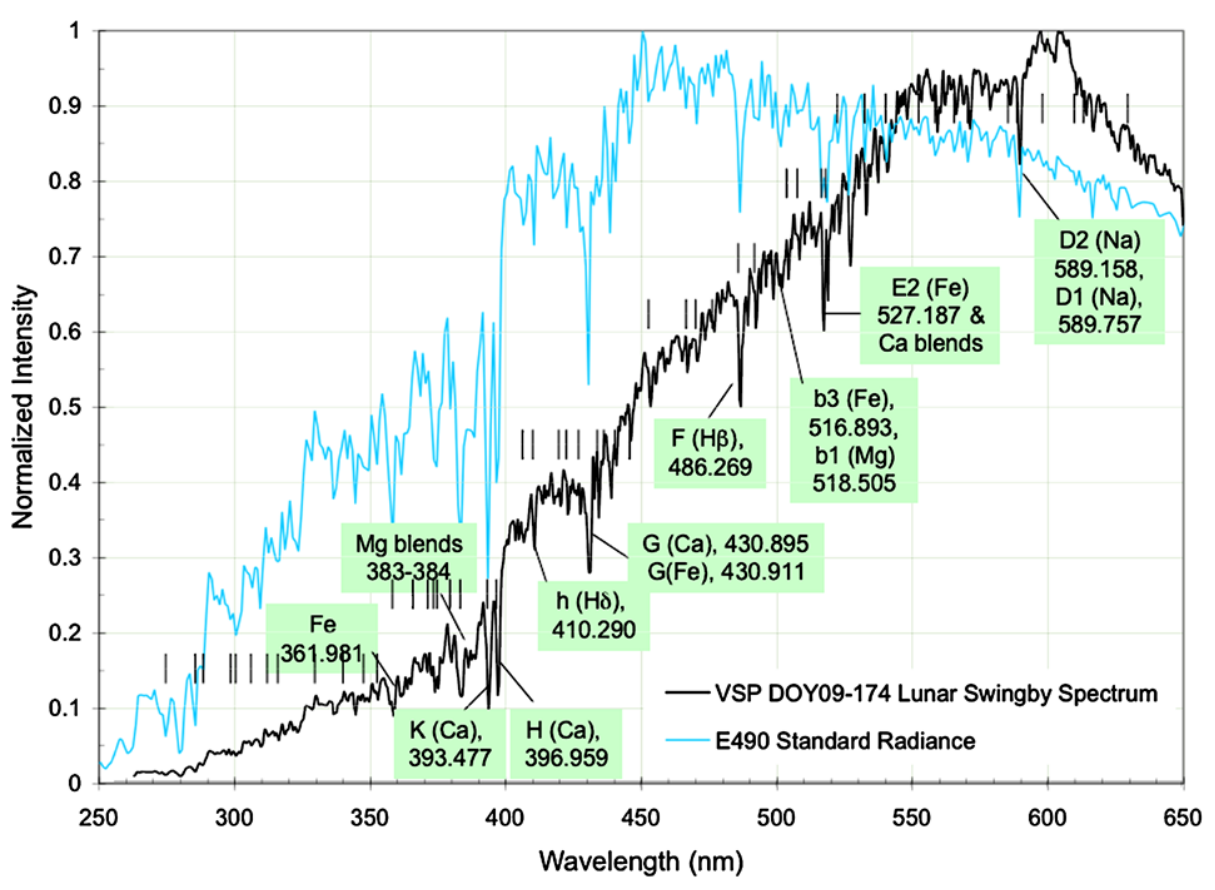

Fig. 11 UV-visible spectrometer (VSP) spectrum from lunar swing-by (black/solid) compared with solar standard (blue/dashed). The strongest lines are identified. Fifty-one solar lines were used for the on-orbit wavelength calibration check and are shown by the vertical tick marks

lines, excluding blends (e.g., some of the brightest Fraunhofer lines and other FeI lines that spanned over several pixels) were identified and assigned wavelengths (in vacuum) from the NIST Atomic Spectral database (Ralchenko et al. 2008). Each line's center location (in pixels) was found by subtracting off the continuum and fitting a Gaussian function to identify the peak location to the nearest half-pixel. A 3rd order polynomial fit mapped the pixel to wavelength relationship. A comparison of the wavelength calibrated lunar swingby VSP spectra to the E-490 zero-airmass standard (ASTM E-490 2000) validated this approach. The majority of the fit residuals (i.e., calculated wavelength-NIST wavelength) were $\pm 0.05 \mathrm{~nm}$, with a peak residual of $0.15 \mathrm{~nm}$. The first and last spectral pixels for the VSP instrument have wavelengths $262.984 \mathrm{~nm}$ and $650.300 \mathrm{~nm}$, respectively. The post-launch VSP calibration also indicated that the VSP had not shifted its wavelength response due to launch, something that had been expected from vibration test history.

Due to schedule constraints, the flight LCROSS VSP unit was not calibrated with a standard radiance source. An engineering test unit (ETU) however, did undergo radiometric calibration using: (1) a NIST-calibrated sphere at the NASA Ames Airborne Sensor Lab (Brown et al. 2005; Cooper 2007) in June 2007 (valid for 380-650 nm) and (2) two UV and visible NIST-calibrated light sources by the vendor in November 2009. In July 2007 there was also a test performed on both flight and engineering test units with a smaller integrating sphere to provide a translation from the NIST-calibrated ETU data to the flight unit, however this technique was only valid for wavelengths $380-650 \mathrm{~nm}$. The derived spectral response for the VSP is shown in Fig. 12. Here the units are defined as DNpR, or "Data Numbers per Radiance," where the radiance units are derived from the NIST sphere, Watts s $\mathrm{s}^{-1} \mathrm{~m}^{-2} \mu \mathrm{m}^{-1} \mathrm{sr}^{-1}$. For the VSP, the DNs represent the instrument's raw response after being dark subtracted 


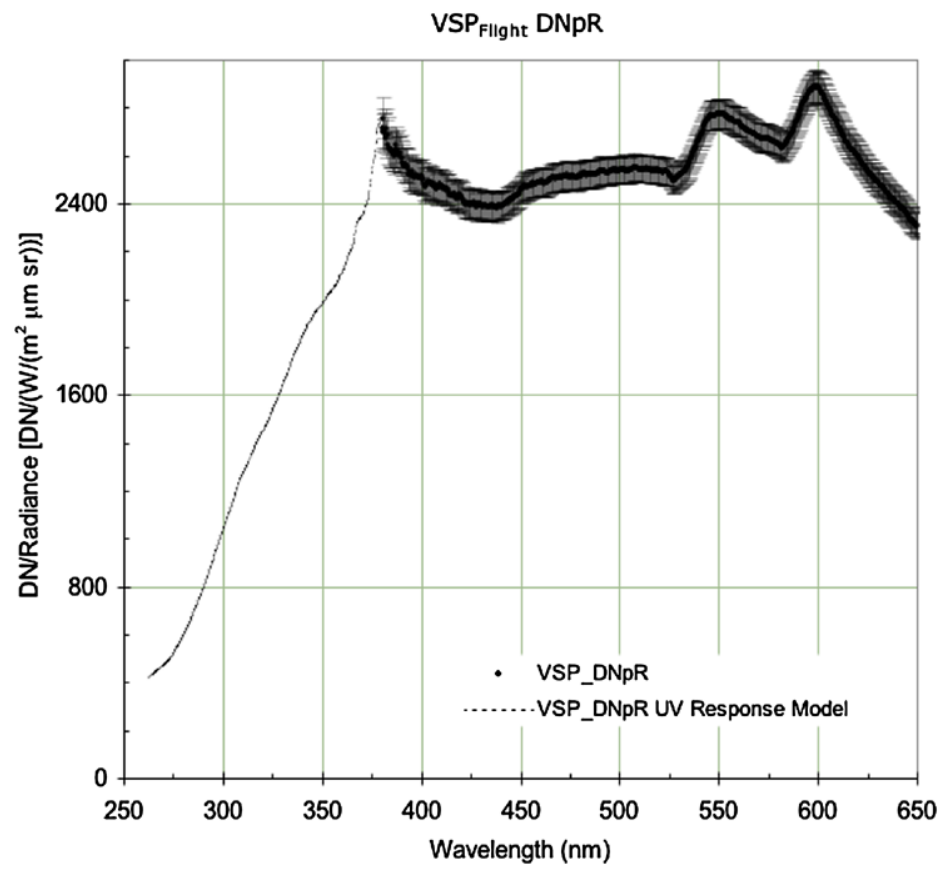

Fig. 12 The spectral response function for the VSP. For wavelength $380-650 \mathrm{~nm}$, the input radiance source used to measure this instrument response was a NIST-calibrated integration sphere. Two spheres were used in the radiance calibration for the flight VSP (see text in Sect. 6.3.1). The error bars are dominated by the spheres' signal variance. In this wavelength region, the instrument's response is relative flat with shape changes attributed primarily to the grating. The fiber and fore-optics are included in this response function. For wavelengths shorter than $380 \mathrm{~nm}$, the VSP was not calibrated. Instead, a model shape was provided by the vendor using a similar instrument and calibrated UV lamp source, no errors provided. This part of the calibration is undergoing validation using flight data and modeling and will be provided in an update to the PDS documentation

and normalized to an integration time of one second. The error bars shown are dominated by the variance of the two spheres used in the calibration. As both spectrometers were sensitive from 250-650 nm, a "best-effort" radiance calibration for 250-380 nm was calculated by normalizing the vendor provided calibration for the ETU $<380 \mathrm{~nm}$ and applying a flight-toETU response model. This part of the calibration is undergoing validation with flight data and modeling and will be provided in an update to the PDS documentation.

\subsubsection{Near Infrared Spectrometers}

For both near infrared spectrometers spectral calibration was performed pre-flight in the NASA Ames Airborne Sensor Lab using a calibrated monochromator (Brown et al. 2005) with a $1 \mathrm{~nm}$ wide slit stepped at $0.01 \mu \mathrm{m}$ increments in September 2008. Hadamard and flash mode spectra were taken at each setting. Each Hadamard/mask transform of the monochromator's $1 \mathrm{~nm}$-wide line extended across several pixels. A Gaussian fit was used to derive the profile's center position (fractional pixel). A 3rd order polynomial fit to wavelength (from the monochromator) versus pixel-fraction (Gaussian-fitted) derived the wavelength calibration. In Hadamard mode, NSP1's first and last spectral pixels have wavelengths $1.169 \mu \mathrm{m}$ and $2.479 \mu \mathrm{m}$, respectively; NSP2's first and last spectral pixels have wavelengths $1.175 \mu \mathrm{m}$ 
Fig. 13 Area-normalized flash mask profiles for NSP1. The masks are labeled M2, M3, M4, M5, and M6, in increasing order of central wavelength. The illumination source was a tungsten lamp within the calibrated ARC monochromator. The lamp's spectral shape declined slowly across the flash mask, so each profile was normalized by dividing by the area under its transmission curve: $\sum[\mathrm{DN}] \times \Delta \lambda / \sum \Delta \lambda$, where $\Delta \lambda$ is in increments of $0.002 \mu \mathrm{m}$. This figure is provided to show each masks' spectral shape and location and relative response within each mask. The spectral shape parameters for each mask are summarized in Table 12

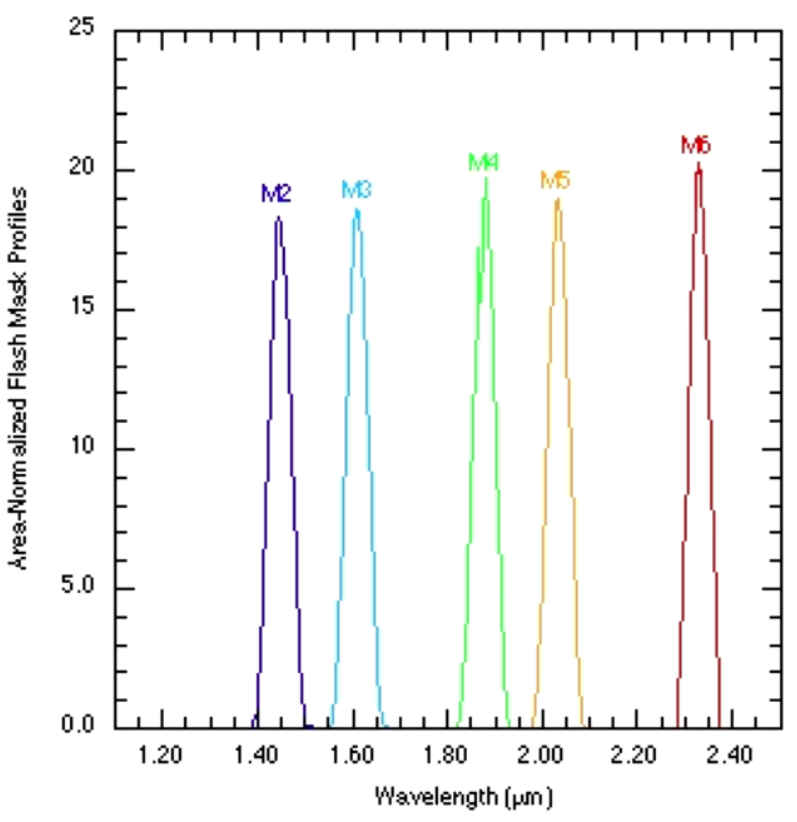

Table 12 NSP1 Flash mode mask central wavelengths and FWHM (full width half max) and FWZI (full width at zero intensity) in microns. The FWZI were approximately $1.35-1.5$ times the FWHM (i.e., the masks profiles are much steeper than the wings of Gaussian profile)

\begin{tabular}{lllllllll}
\hline Mask $^{\mathrm{a}}$ & FWZI_ctr & FWZI_start & FWZI_end & FWZI & FWHM_ctr & FWHM_start & FWHM_end & FWHM \\
\hline M2 & 1.448 & 1.394 & 1.502 & 0.108 & 1.447 & 1.420 & 1.474 & 0.054 \\
M3 & 1.610 & 1.556 & 1.664 & 0.108 & 1.610 & 1.584 & 1.636 & 0.052 \\
M4 & 1.877 & 1.824 & 1.930 & 0.106 & 1.877 & 1.852 & 1.902 & 0.050 \\
M5 & 2.032 & 1.978 & 2.086 & 0.108 & 2.034 & 2.008 & 2.060 & 0.052 \\
M6 & 2.329 & 2.276 & 2.382 & 0.106 & 2.331 & 2.308 & 2.354 & 0.046 \\
\hline
\end{tabular}

a Mask 1 is a dark mask defined as all MEMS mirrors in an 'off'/dark position. The dark mask value is subtracted from each intensity mask value to remove drifts

and $2.479 \mu \mathrm{m}$ respectively. Instrument spectral resolution, defined here as FWHM width, was derived by multiplying the Gaussian-fit width (pixel-fraction) by the first derivative of the wavelength equation $(\mathrm{FWHM}(\mu \mathrm{m})=\mathrm{FWHM}(\mathrm{pix}) \times d \lambda / d x(\mu \mathrm{m} /$ pixel $))$. A FWHM measurement was composed of samples taken at 30 spectral positions. The technique was repeated at a finer $0.002 \mu \mathrm{m}$ increment to measure instrument profiles. NSP1 line profiles at 1.480 and $2.064 \mu \mathrm{m}$ had FWHM 0.034 and $0.036 \mu \mathrm{m}$, respectively. NSP2 line profiles at 1.378 and $2.072 \mu \mathrm{m}$ had FWHM 0.033 and $0.032 \mu \mathrm{m}$, respectively.

The LCROSS NSP Flash mode design had five spectral masks and one dark mask per scan. The masks were chosen to sample in and outside the water-band regions at $\sim 1.4$ and $\sim 1.8$ microns. The dark mask was defined with the MEMS mirrors in an 'off' state to measure the intrinsic thermal background of the single-element detector and internal scattered light contributions. Area-normalized flash mask profiles for NSP1 are shown in Fig. 13, their key parameters summarized in Table 12. For these measurements, the ARC Monochroma- 
tor was stepped $0.002 \mu \mathrm{m}$ over a range of wavelengths sufficient to map each mask profile. The Flash $(72 \mathrm{~Hz})$ mode on LCROSS was a unique operation for these spectrometers and provided the solution for rapid sampling required for the expected impact flash, whose near infrared duration was predicted to be $200 \mathrm{~ms}$ (actual flash was detected by NSP1 in Flash mode had a rise time of $110 \mathrm{~ms}$ and was well-sampled, see Schultz et al. 2010). In comparison, Hadamard (full-spectrum) mode, the instrument provided lower resolution spectra over $1.2-2.4 \mu \mathrm{m}$ at a lower $1.7 \mathrm{~Hz}$ cadence.

Unlike the UV-visible spectrometer, both near-infrared spectrometer flight units did undergo radiometric calibration using the NIST calibrated sphere at NASA Ames Airborne Sensor Lab (Brown et al. 2005; Cooper 2008) in September 2008 with measurements valid for the entire 1.2-2.4 $\mu \mathrm{m}$ wavelength range. The approach to the radiance calibration of the near infrared spectrometers was methodical but performed in steps over several test dates. The steps were necessary since the flight units in their actual flight configuration (in particular, flight fiber length and bend geometries) were not available for direct calibration with the NIST sphere. To assess the quality of the systematic errors in the use of the NIST sphere, a detailed study was performed on the NSPs, where the location of the fore-optics to sphere center was adjusted by a few centimeters vertically and horizontally, different test fibers were used, test fibers were bent with one and two bends, and various baffling schemes were introduced. Assuming the sphere remained constant, as it should have, variances in the detected signal could be computed. The data obtained showed that systematics introduced at most $\sim 4 \%$ error, with samples shortward of $1.2 \mu \mathrm{m}$ and longward of $2.1 \mu \mathrm{m}$, subject to the largest error, due to the poor response of either the instrument or the lamp, respectively (Wooden et al. 2009). The NIST Sphere itself was quoted to provide $2.2-3.7 \%$ error, wavelength dependent (Cooper 2008).

It was observed early in the mission, during lunar swingby (DOY09-174), that applying the September 2008 lab-based radiometric calibration relationships for the two near infrared spectrometers introduced "water emission features" in their responses. It was then realized that the lab-based radiance calibration provided a 1-meter long path length that introduced water absorption that was not fully accounted for by the provided NIST radiance curve. The lab-based testing had not been performed in a vacuum, a test oversight, and the NISTprovided spectrum would appear to have its own undocumented water contamination. The LCROSS payload team devised a series of calibration steps using flight NSP2 data to correct this issue. The steps are discussed below with key points summarized in Figs. 14 through 18.

The solar-viewing near infrared spectrometer (NSP2) contained a diffuser as its foreoptics. This instrument obtained solar spectrum at a range of incident angles with a response that closely followed a typical cosine, particularly for incident angles less than $50^{\circ}$ from normal. High signal-to-noise ( $>10^{6}$ counts) spectra was obtained on three observations: lunar Swingby (DOY09-174), EarthCal2 (DOY09-229) and the final descent (DOY09-282), where peak signal was measured at solar angles $14.0^{\circ}, 40.2^{\circ}$ and $12.6^{\circ}$, respectively. A comparison of the solar spectra measured for these observations with the solar standard (E-490, 2000) is shown in Fig. 14a. In other flight observations, the NSP2 was aligned at solar angles $80-90^{\circ}$ providing poor signal-to-noise spectra to be useful for calibration. A careful inspection of the high signal-to-noise NSP2 solar spectrum was performed to rule out contamination by hydrazine, the propellant used for attitude control during instrument operations, and ammonia, its primary decay product. No contamination was seen in the data from the missions' final hour. This spectrum was used to correct the lab-based NIST calibration radiance for the flight NSP1 instrument. The observed solar spectrum was featureless at this spectral resolution $(R \sim 40-80)$. Therefore a post-launch wavelength calibration check could not be directly performed. The wavelength calibration for this instrument was derived from the pre-launch baseline. 
Fig. 14 (a) Solar spectra measured by NSP2 for three different solar angles during flight (solid black

circles/Swingby; solid red triangles/EarthCa12; open black squares/Preimpact-Impact). The solar standard radiance (E-490) is shown by the dashed light-blue line. (b) Flight NSP2 computed response function (units DN per Radiance) using the mission's last day measurements when contamination was assumed to be minimal. NSP2 has its peak response at $\sim 1.7 \mu \mathrm{m}$. The error bars are NSP2's signal variance in 10 spectra. This is the NSP2 spectral response function delivered to the PDS
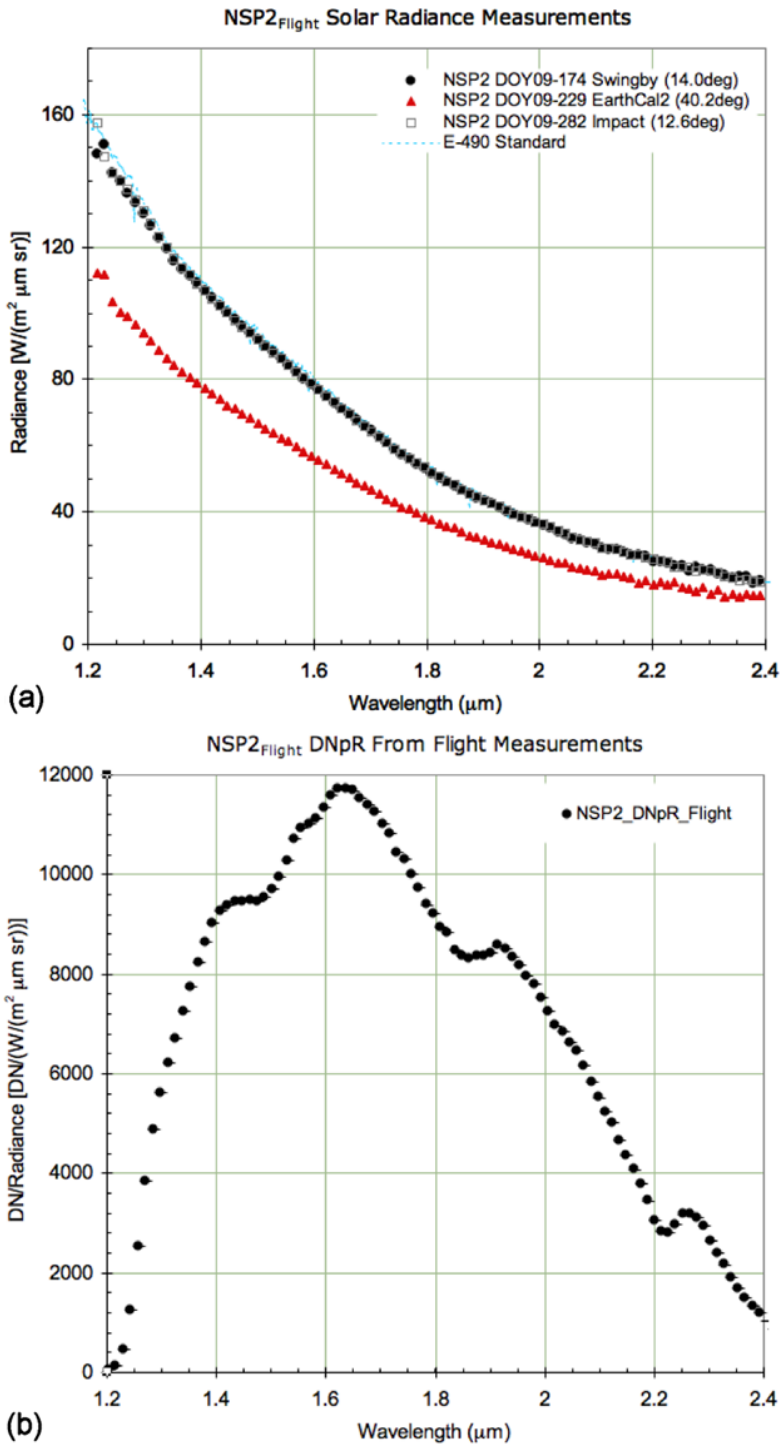

The flight NSP2 radiance calibration was derived directly from the solar data, shown in units DNpR, or per or "Data Numbers per Radiance" units, where the radiance units are that of the NIST sphere, Watts s $\mathrm{s}^{-1} \mathrm{~m}^{-2} \mu \mathrm{m}^{-1} \mathrm{sr}^{-1}$ (Fig. 14b). This is the calibration provided to the PDS.

Both the NSP1 and NSP2 underwent radiance calibration in the lab using a NISTcalibrated sphere, whose spectral shape and assigned variance is shown in Fig. 15a. In both cases the units were tested in the "NSP1 configuration", i.e. a single $75 \mathrm{~cm}$ length fiber and the $1^{\circ}$ diameter fore-optics. A comparison of the two instrument's response functions in this arrangement are shown in Fig. 15b. As a result of this test, NSP1, the more sensitive instrument, was chosen as the nadir spectrometer. 
Fig. 15 (a) Radiance vs. wavelength for the NIST-calibrated integrating sphere. This sphere was the illuminating source during lab-based radiometric calibration of the UV-visible and near-infrared spectrometers. The error bars are the sphere's signal variance as supplied by the NIST report. (b) Pre-flight spectral response functions (units DN per Radiance) for NSP1 (solid black circles) and NSP2 (open black circles) computed from the lab NIST-calibrated integrating sphere. NSP2 shown here is in "a NSP1-configuration" (i.e., has a $1^{\circ} \mathrm{FOV}$, no diffuser, and $75 \mathrm{~cm}$ length fiber). Both spectrometers' peak responses are at $\sim 1.7 \mu \mathrm{m}$, and they show spectral differences. NSP2 could not be tested with the NIST sphere in its flight configuration because its $130^{\circ} \mathrm{FOV}$ was too large for the sphere's exit aperture. The errors shown are those computed from the weighted mean of the measurements and the sphere's variance
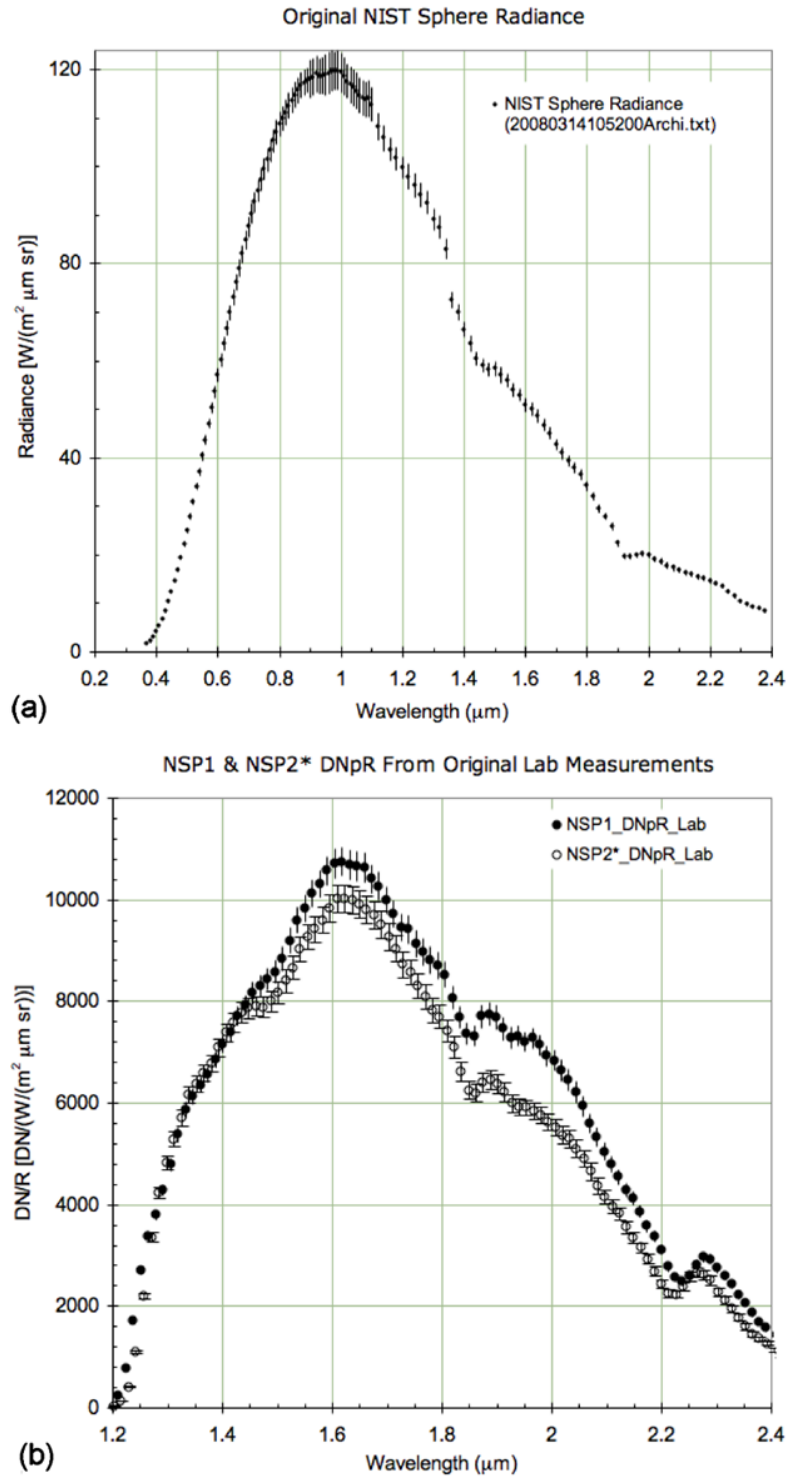

Converting to a NIST sphere lab setup configuration was necessary for the watercorrection to the NSP1 lab-calibration response function, therefore NSP2's response function, derived in flight, had to first be adjusted to match the former. Figure 16a shows the wavelength dependence of these corrections. Figure 16b illustrates how these corrections affected the NSP2 spectral response function. After these corrections were applied, a direct comparison to the NSP2 response function, derived from flight, could be compared to its pre-flight lab measurements. This is shown in Fig. 17a. The ratio between the two NSP2 sphere response function provided the correction.

Finally, Fig. 17b shows the NIST sphere radiance as a function of wavelength for the original NSP2 lab measurement and that derived from NSP2's flight measurement. Deeper 
Fig. 16 (a) The following corrections were applied to the flight NSP2's response function derived from the sun data (see Fig. 14b) with its pre-flight NIST-sphere data (open black circles in Fig. 15b). The corrections were: (1) bare fiber to the $1^{\circ}$ FOV scope fore-optics (red/solid squares),

(2) attenuation due to different fiber lengths (145 cm to $75 \mathrm{~cm}$ lengths) (orangelopen squares), (3) slope variations due to fiber bend configurations

(yellow/closed triangles),

(4) transmission from NSP2's

two 1-mm thick sapphire

windows (green/open circles),

(5) transmission from

$0.01778 \mathrm{~mm}$ thick NSP2

Spectralon diffuser to bare-fiber (dark bluelclosed diamonds),

$\left(5^{\prime}\right)$ applying a scalar correction to match peak NIST-sphere radiance (no slope or feature changes) (light bluelopen diamonds). The measurement errors associated with each correction are shown (black markers) but are smaller than the symbols in these plots.

(b) Application of the correction factors summarized in (a) to the flight NSP2's response function. The intra-step color/symbol designations are the same as for (a). The input spectrum (black/solid circles) is the in-flight measured NSP2 response function from the Sun (same as the solid black circles in Fig. 15b). The final spectrum (light bluelopen diamonds) is the computed response function for NSP2 in a NIST-sphere lab setup
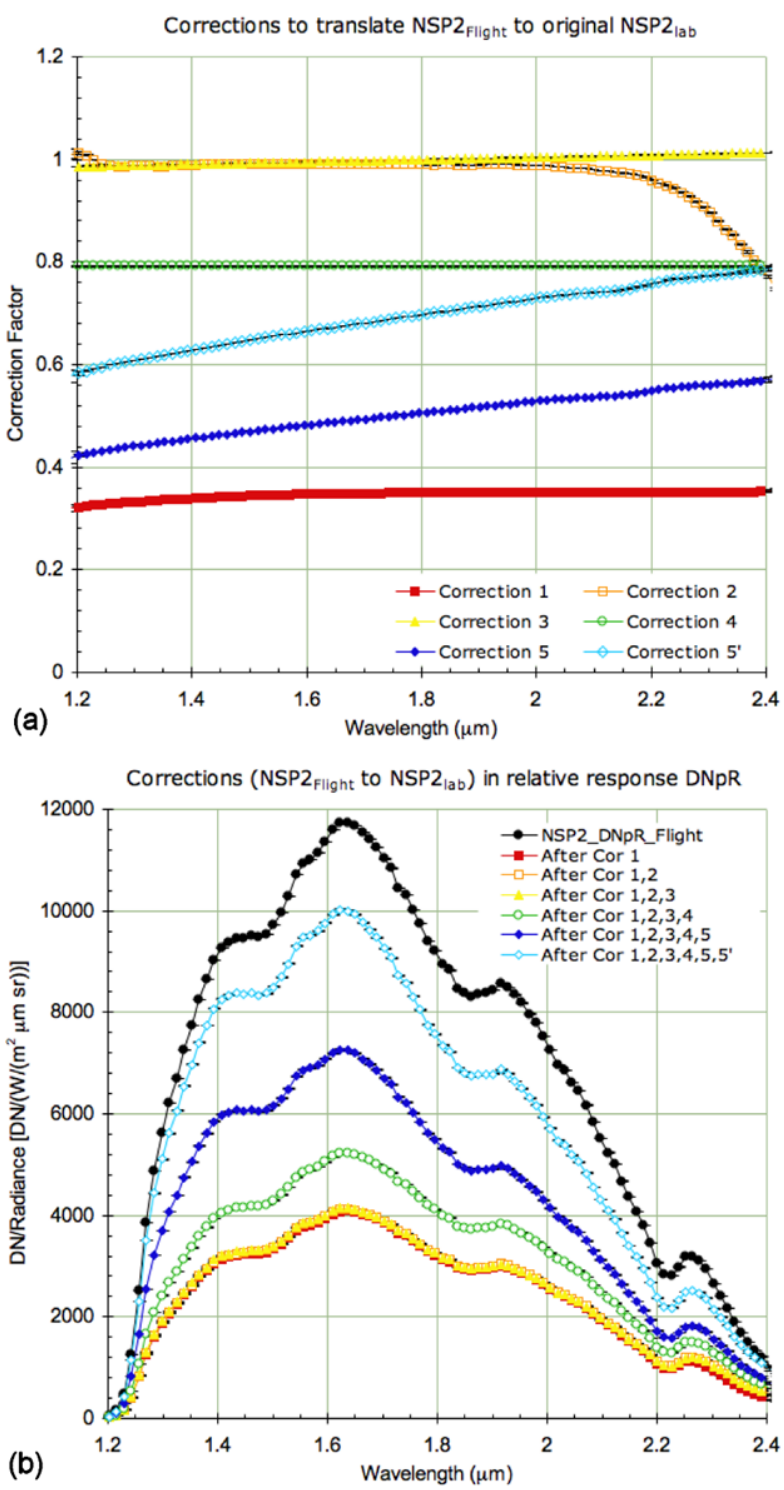

water absorption features are seen in the former, which is understood to be due to the 1 meter path length in the ARC calibration lab. This "water-adjusted" radiance. or more correctly, a "water-enhanced" NIST sphere radiance, is the radiance used to derive the flight NSP1 radiance calibration (Fig. 18a). NSP1 was also used in flight in Flash mode. The radiance calibration for the five spectral masks are shown in Fig. 18b. These two calibrations were provided to the PDS.

The NSPs are Hadamard transform spectrometers. They introduce noise when the source varies quickly relative to the time over which spectra are captured. This occurs because these spectrometers sample different frequencies at different times while collecting data for a single spectrum. This noise can be corrected for by estimating the change in scene 
Fig. 17 (a) Summary of NSP2 response functions used for the correcting water contamination in the NIST-sphere radiance. The direct solar NSP2 flight measurement is shown in black/solid circles (same as in Fig. 14b) and the measurement-corrected for fore-optics and fiber differences to match the lab-based set-up is shown in light-bluelopen diamonds (same as in Fig. 16b). The original NSP2 pre-flight lab response function is shown as blacklopen circles (same as in Fig. 15b). The ratio of the light bluelopen diamonds to black/open circles is the correction to be made to the sphere radiance to account for water in the pre-flight lab calibration functions. (b) The NIST-sphere spectral radiance functions for the two cases in (a). The solid black circles/black line is the original pre-flight sphere spectrum (same as in Fig. 15a). The open blue circles/blue line is the "water-adjusted" NIST sphere radiance to be used for calibration of the flight NSP1 spectra. This "additional" water is attributed to the unique path length in the ARC calibration lab
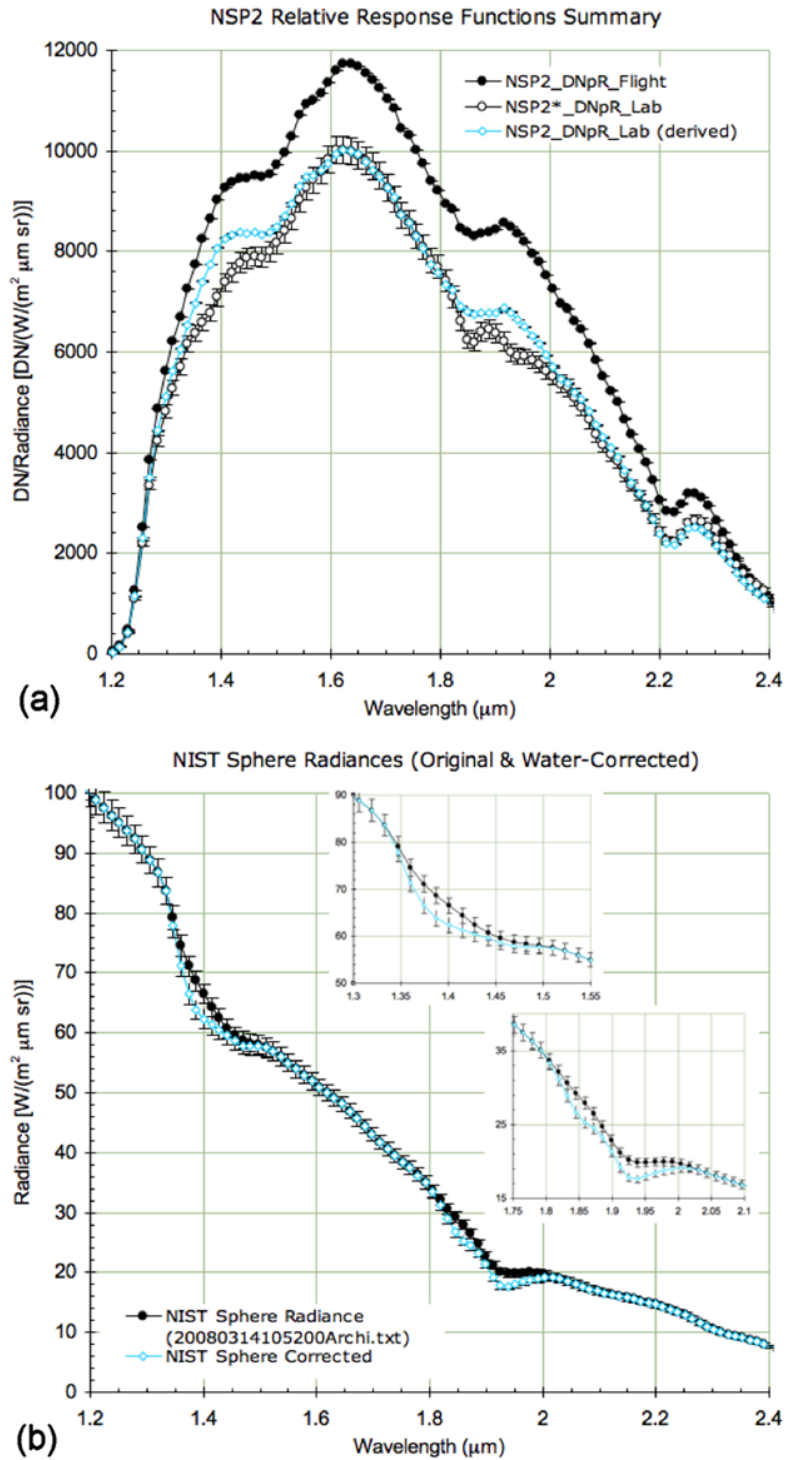

brightness between each spectrum and the next, assuming this change occurred linearly over the 0.6 second data collection period, scaling the raw, pre-Hadamard transform data for each spectrum to reflect the brightness at the beginning of data collection, and re-processing the data to generate a corrected, calibrated version of it. We applied this approach to NSP1 spectra obtained during the final minutes of the mission. The estimate of scene brightness for each spectrum was done first by adding up total radiance and again by sampling the raw data during a 28 millisecond period at the beginning of each data acquisition. These two methods produced very similar corrections.

For the LCROSS data, we have determined this noise to be below $0.5 \%$ between 1.3 and $2.1 \mu \mathrm{m}$. For wavelengths longer than $2.1 \mu \mathrm{m}$, these instruments have a very poor response function (e.g., see Figs. 14b, 15b and 18a). Figure 19 shows the ratio between a corrected 
Fig. 18 (a) The flight NSP1 computed response function (units DN per Radiance) calculated from applying the "water-adjusted" NIST sphere radiance (blue line from Fig. 17b) and its errors. This is the NSP1 response function delivered to the PDS. (b) NSP1 Flash mode response function is computed by convolving the NIST Sphere radiance values with the derived instrument profile for each of the masks (see Fig. 13). Shown here are the pre-flight lab (open blue circles), and the flight NSP1 computer response functions after applying the same adjustment as required for Hadamard mode (solid black circles). The NSP1 mask response function delivered to the PDS are the solid black circles.

The horizontal lines here represent the FWZI extent of the masks (see Fig. 13)
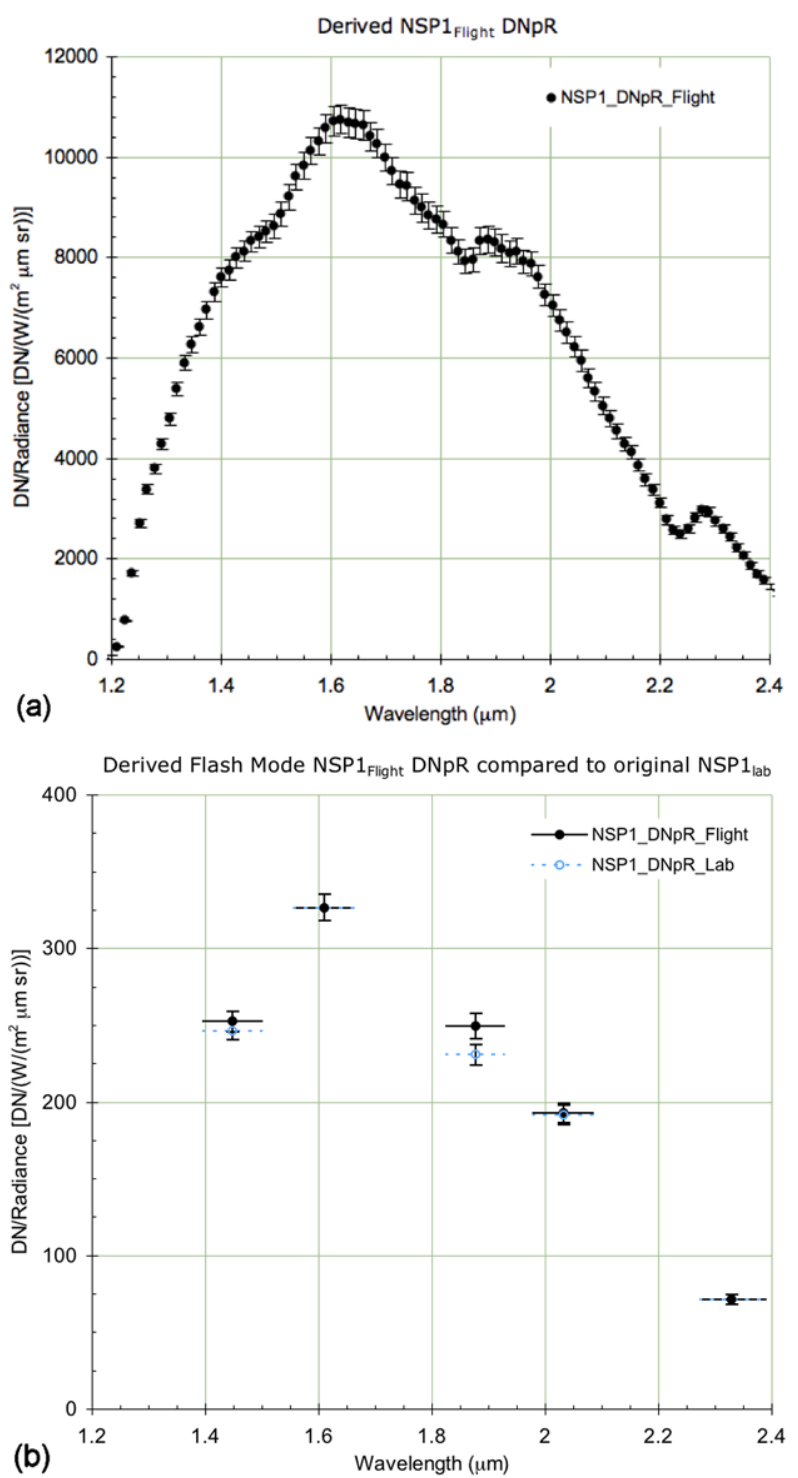

average of 133 NSP1 (nadir) pre-impact spectra and the equivalent, uncorrected average. Ratios of corrected and uncorrected post-impact spectra were similar. This assessment was computed after the LCROSS data was delivered to the PDS and is not reflected in that dataset.

\subsection{Near Infrared Cameras Calibration}

In operation, the near infrared cameras had a single changeable parameter: the camera OPR (Operational Configuration). This OPR, ranging from 0-15, which although represented by one parameter, changed two settings: exposure integration time and internal gain. This 


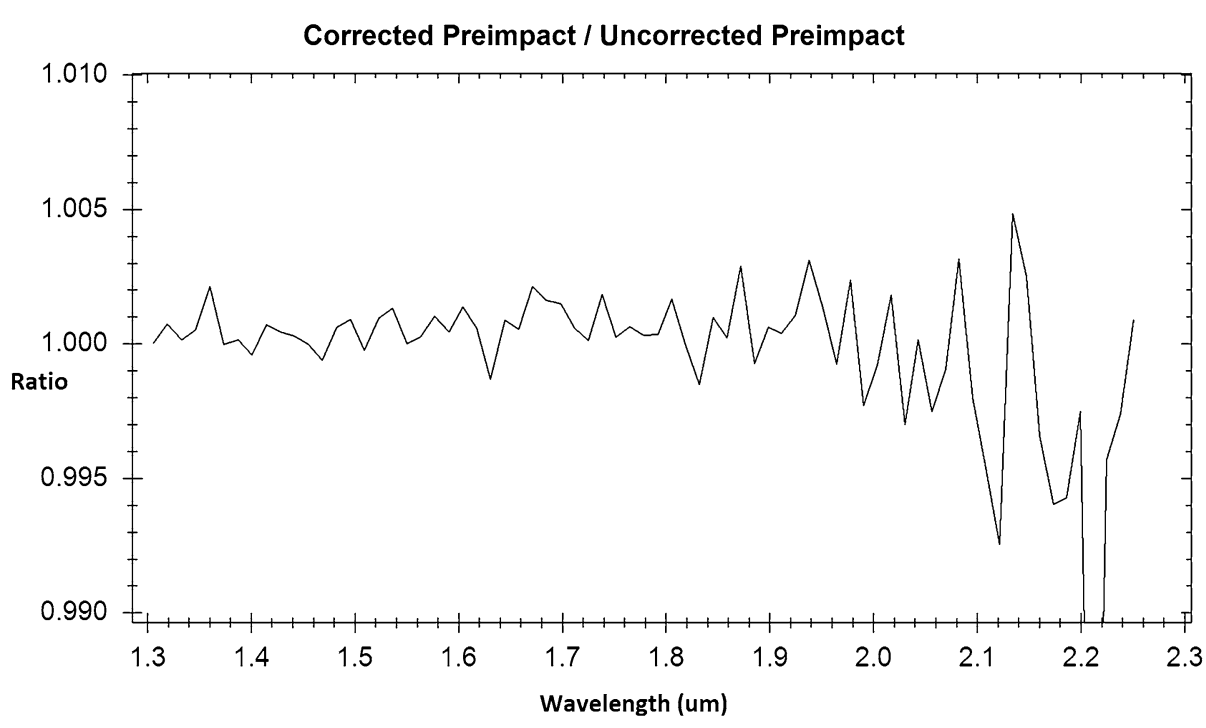

Fig. 19 This plot illustrates noise in NSP1 spectra introduced by varying source brightness. 133 pre-impact or reference spectra were averaged both with and without the correction described in the text, and then ratioed. This ratio remains between 0.995 and 1.005 ( $0.5 \%$ variation) between 1.3 and $2.1 \mu \mathrm{m}$

behavior was known before launch and was measured in the lab using a series of filled-FOV flat-field type images of different illuminations.

Because the lunar swingby sequences for these cameras had been preloaded before launch and the moon's phase and illumination (as seen by the LCROSS spacecraft) during swingby was not known until the launch date, a decision was made to put the near infrared cameras into an autogain mode for the swingby and tie on-board performance to the lab test data. In practice, this failed to work. At altitudes $\sim 8,000-10,000 \mathrm{~km}$ during the actual June 22, 2009, swing-by, the bright moon filled less than half of the scene (Fig. 20). This caused the cameras to overestimate the exposure since a majority of the pixels were not illuminated. The moon was therefore saturated at these longer exposures and no valid on-orbit images were obtained during swingby for the near infrared cameras, except two NIR1 camera images, the result of identifying the problem during flight and attempting exposure changes in real-time. At the time of swingby, the LCROSS payload also experienced an issue with decompression algorithms and lagged in processing (see below), so live attention and attempts at real-time commanding was also being made to diagnose the problem during a limited operational time ( $<20$ minutes) window.

To address this early lack of on-orbit near infrared camera performance validation, a special exposure test was designed for the Earth-look calibrations. Because the camera settings needed to be preloaded on the DHU and had to fit within a 29 kbps bandwidth and within a planned spacecraft absolute time sequence, a selection of OPRs were used, chosen to bracket the bright Earth radiance. Typically, for these cameras, low OPR values (2-4) provided non-saturated images for Earth scenes (Fig. 21).

The lunar scene during impact, however, would be much fainter than the bright Earth. A correlation between the nadir near infrared spectrometer's (NSP1) signal and the two NIR cameras, using data from both Earth calibration data and laboratory scenes, was established to provide a look-up table for potential future NIR camera setting changes. This approach 

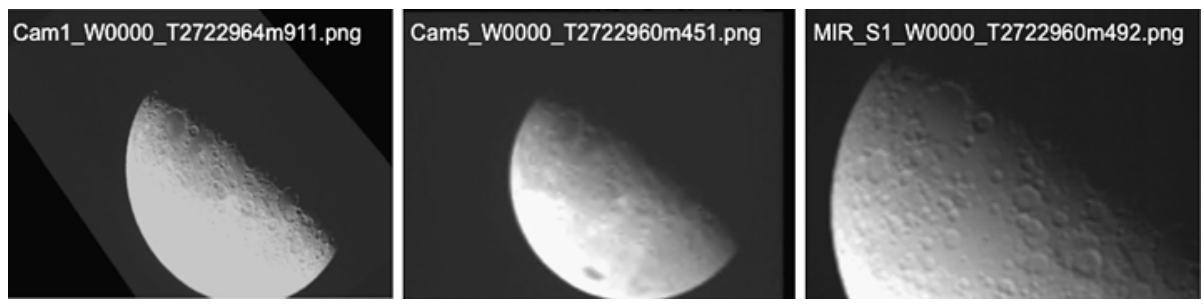

Fig. 20 Single unprocessed images taken (left to right) VIS/Cam1, NIR1/Cam5 (1.4-1.7 $\mu \mathrm{m})$ and MIR1/MIR_S1 $(6-10 \mu \mathrm{m})$ during lunar swingby (DOY09-174) target 2 measurements and rotated to align to same view. The FOVs are $30.1^{\circ} \times 22.8^{\circ}, 28.7^{\circ} \times 21.7^{\circ}$, and $15.0^{\circ} \times 11.0^{\circ}$, respectively. LCROSS was $\sim 8,800 \mathrm{~km}$ altitude from the Moon at this point in the swingby. NIR1 is slightly out of focus, a known issue prior to launch
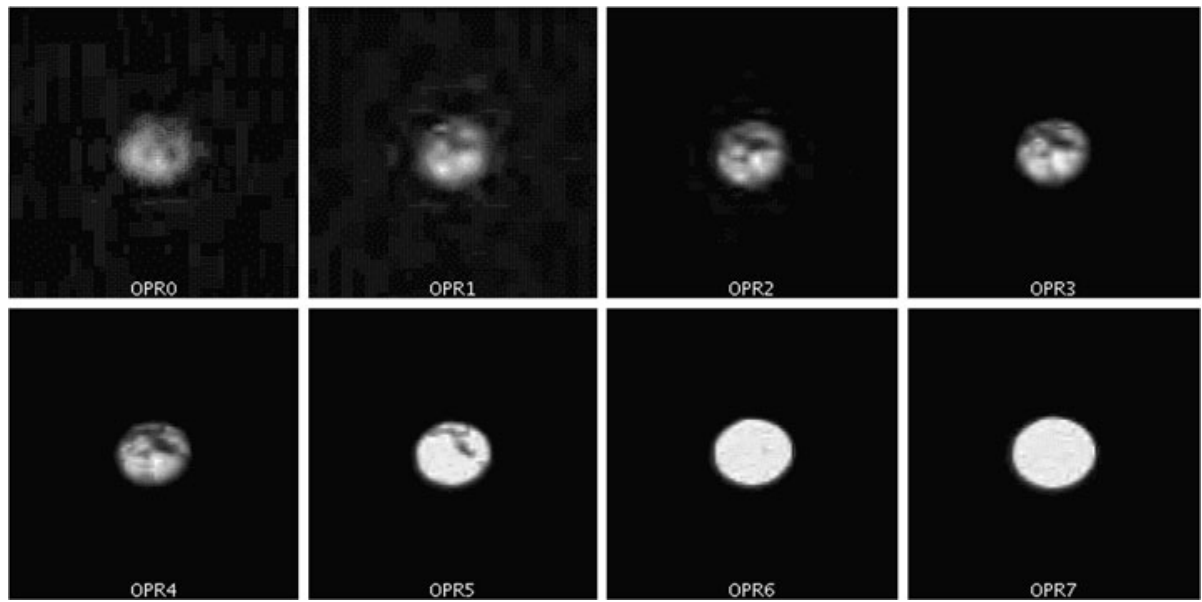

Fig. 21 NIR2 Exposure time test during DOY09-213 Earth calibration. The camera took images of the Earth at OPR settings 0 through 7. The images below are shown on the same linear scale. For this dataset, NIR2 saturated for OPR $\geq 5$. A similar test was performed for NIR1 (data not shown)

had the caveat that the NSP1 only probed the center one degree of the $\sim 30$ degree FOV of the NIR cameras.

With this analysis, for the final hour of the mission, NIR1 and NIR2 were set for OPR 7 and OPR 5, respectively, to stay within the linear range of the NIR camera response for the lunar South Pole scene and not experience blooming, a condition where a saturated subsection leaks additional signal into adjacent pixels, and in most severe cases, spreads across the entire array. These settings proved to be optimal. The small 8-bit range of these camera and saturation effects that spread across the array truly limited the camera's performance in a scene of a wide range of illuminations. As a result, to prevent loss of data due to saturated pixels that would bleed into adjacent pixels, a delicate balance was needed. In the final minutes of the mission, the unexpected faintness of the ejecta cloud indicated that the cameras had underexposed on the cloud. Applying aggressive image stretching, the plume was barely detected in the NIR cameras, but not with enough quality to be used for ejecta plume size measurements (Schultz et al. 2010). Fortunately, the visible camera was able to observe the cloud in multiple images and spectroscopic information about the ejecta 
was successfully measured by the visible and near infrared spectrometers in the final four minutes (Colaprete et al. 2010).

The unfiltered camera (NIR2) was later adjusted to OPR 10 (longer exposure) in the final few frames of the mission when the camera was observing a darker scene, the bottom of the Cabeus crater. Originally the models predicted radiances from the bottom of this permanently shadowed crater to require longer exposures. In practice, scattered light from the adjoining crater walls, although out of view, and perhaps thermal contributions from the newly-created created, although on a smaller spatial scale, added unexpected signal. After successfully shortening the exposure time, in near-real time, ten good quality images of the crater floor were collected. These images led to the identification of the Centaur-impact crater and ejecta cloud seen from $<35 \mathrm{~km}$ altitude (Schultz et al. 2010).

Calibrated NIR camera products for the PDS are based on testing using a flight spare camera (including a flight spare $1.4 \mu \mathrm{m}$ long-pass filter for the filtered (NIR2) flight camera configuration). In this test the camera-recorded integrated signal from the NIST-calibrated sphere, the same sphere used for the spectrometer testing (see Sect. 6.3), but since the NIST radiance was too bright for these cameras, external infrared neutral density filters were used to attenuate the incident light. The integrated NIST radiance, attenuated by the neutral density filters and convolved with a water-correction (derived from the NSP lab measurements (see Sect. 6.3.2) and extrapolated down to the 0.9-1.7 $\mu \mathrm{m}$ range), was computed for the pixels in each camera's response range. The aim for this best effort approach, described above, was to assess order of magnitude settings in case on-the-flight exposure changes were made, which did happen on the final descent.

\subsection{Thermal Camera Responsivity Drifts}

Pre-launch thermal vacuum data indicated that thermal camera response to a fixed scene was expected to drift with time. This was common to both cameras up to 600 seconds since power-on, but MIR2 continued to drift beyond 50 minutes after power-on. It should be noted that for data taken in atmospheric pressure, both cameras also showed a "power-on" transient, but then settled to a normal response after $\sim 600$ seconds. To illustrate the vacuum-drift issue for MIR2, Fig. 22 shows the two thermal cameras' mean value for the first 20 minutes after power-on for three data sets taken at vacuum. Two data sets are from flight and one is from thermal vacuum testing in May 2008. The mean value for the entire image is plotted, whose absolute value is strongly dependent on the scene (e.g., moon filling scene as in pre-impact/impact or empty sky like in starfield or separation), and thus an offset was added to bring the data on to the same scale for a comparison with time. The cameras' operating temperatures were essentially the same for all operations, removing that aspect. Because the scene was not changing spatially (and not expected to be changing thermally either) the mean camera response was expected to be flat, with occasional expected artifacts due to flat-fielding every two-minutes. MIR1 demonstrated a transient response within the first 600 seconds after power on, after which remained consistent with expected nominal behavior. MIR2 did not. The FOVs of the two cameras overlapped sufficiently that both cameras observed the same scene. The longer-time temporal drift in MIR2 response in vacuum continues to be an issue for providing a valid calibration to temperature response for it. This behavior of the FLIR camera in vacuum showed that the packaging provided by the Thermoteknix vendor did provide sufficient stability used for the internal flat fielding calibration.

Despite this behavior in vacuum, both thermal cameras were able to detect the thermal signature of the newly formed crater in several images. This was essential for identification 

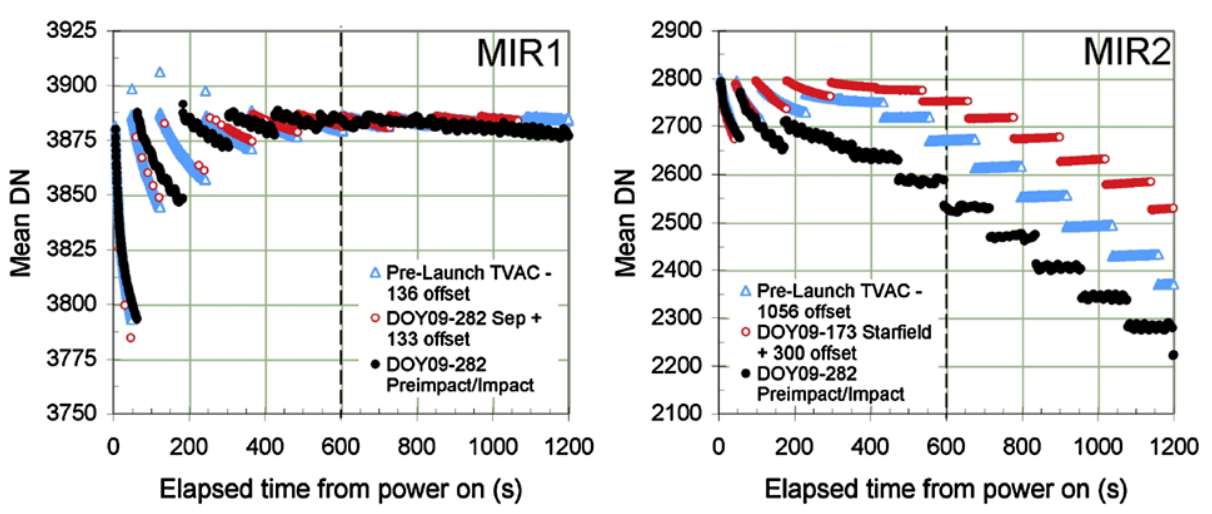

Fig. 22 Mean value (scene will affect overall level) for first 20 minutes for vacuum data for (left) MIR1 and (right) MIR2. The discrete jumps are due to known flat field corrections occurring every 120 seconds. The vertical line is placed at $600 \mathrm{~s}$ from power on, where the MIR1 data flattens in response (with a constant scene). The MIR2 image, however, continued to drift. In all measurements, both cameras observed the same temperature scene and should have shown similar time dependent trends

of the crater's location and size, the former to validate mission pointing requirements and the latter to constrain impact models. The temperature calibration for MIR1 during the final hour, which is applicable to both the curtain and crater observations, is discussed in more detail below. MIR2's temperature calibration is highly uncertain due to unique drifts in the final hour that did not match previous testing trends.

MIR1 temperature calibration was derived from a comparison with a thermal model provided by LRO Diviner (Paige et al. 2010a) team for the lunar South Pole at the day and time of the LCROSS impact (DOY09-282, 11:31 UTC). This model took into account the infrared emissivity and solar reflectance of the lunar surface and had been validated against actual Diviner measurements. For temperatures below $200 \mathrm{~K}$ and above $350 \mathrm{~K}$, the model agreed with Diviner flight data to $\pm 2 \mathrm{~K}$ and $\pm 5 \mathrm{~K}$, respectively (Dave Paige, priv. communication). The pixel width for the Diviner image was based on SELENE data, $\sim 1 \mathrm{~km} / \mathrm{pixel}$. At $599 \mathrm{~km}$ altitude (time of impact), the LCROSS MIR1 \& MIR2 had $\sim 1 \mathrm{~km} /$ pixel footprints. The pixel scale is approximately the same for the two images, minimizing sub-pixel mixing.

Thirty-one sub-arrays, chosen because of their location relative to prominent features (e.g., hot crater rims, lower temperature flat expansive areas) that was recognizable in the MIR1 data, were used to sample a range of temperatures from the Diviner model (in degrees K). A comparison for the Diviner model and the LCROSS MIR1 image (at the time of the impact flash, circled) and the 31 selected samples (numbered squares) is shown in Fig. 23. It should be noted that the sampled areas specifically did not include data from the coldest regions $(<220 \mathrm{~K})$, for which the camera's low temperature performance was most uncertain. MIR1 and MIR2 consistently could not distinguish values $<200 \mathrm{~K}$, identifying a low temperature limit sensitivity for these cameras in operation. A 3rd order polynomial fit provided a translation from MIR1 raw counts (DN) to temperature (degrees Kelvin). Application of this calibration to MIR1 images taken at 253.4 seconds after impact revealed a warm crater temperature (it was the only 'hot' crater in the $15^{\circ} \times 11^{\circ}$ camera field of view), spatially spanning $\sim 3$ pixels diameter at spatial resolution $9.5 \mathrm{~m} /$ pixel, but at the limit of the camera's thermal sensitivity. 


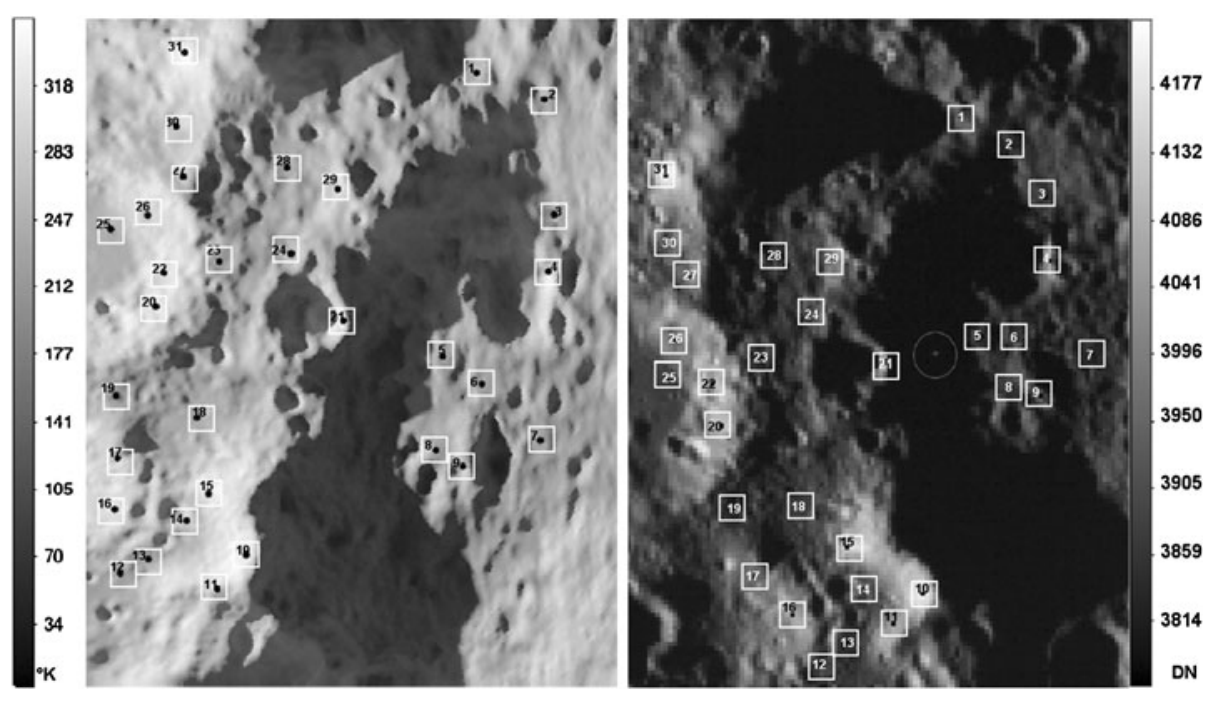

Fig. 23 (Left) Diviner thermal model image in greyscale of the Cabeus crater, where values are in Kelvin (see text for model description). Crater rims are $\sim 310-330 \mathrm{~K}$, whereas the Cabeus crater bottom is $\sim 30-70 \mathrm{~K}$. (Right) Raw LCROSS MIR1 image at time of impact, values shown as Data Numbers (DN). The Centaur impact crater's thermal signal is circled, registering above the cold crater basin. In both images, the black dots, centered within the numbered squares, represent sample points used for the mapping, using the terrain as an identification guide

\subsection{Scene Complexity and Bandwidth Limitations During Lunar Swing-by and the Final Descent}

One of the challenges in designing the impact event flight sequence was to optimize the near-simultaneous sampling of multiple instruments and fit within the allocated bandwidth. On LCROSS, to fit within the $1 \mathrm{Mbps}$ data rate limitation for the science downlink during the mission's final hour, a lossy compression algorithm that typically reduced each image to $1 / 20$ th its original size was used. Lossy methods achieve greater compression than lossless methods by actually removing parts of each image. The algorithm tried to find subtle details whose removal the human eye would not notice. Being able to combine many different kinds of data into a single digital data stream is so useful that this approach has been standard practice for many years.

The complexity of the lunar scene was underestimated during ground testing. Prior to launch, most image-size testing was performed with a large reproduction of the moon's pole in front of the cameras. This, however, did not mimic the high contrast and detail of the real scene, particularly for the thermal cameras. Scene complexity mattered because the images were highly compressed and changes in the actual lunar scene easily changed the sizes of the compressed images by a factor of 4 . This behavior was first observed during the lunar Swingby in the first week of the mission. With increased image sizes by all five cameras, particularly a factor of 4 in each of the thermal cameras, the designed on-board swingby sequence provided more data than could be handled by the $1 \mathrm{Mbps}$ bandwidth allocation and subsequently, images, mostly visible and NIR, were dropped in the data stream, based on instrument priority in the DHU. Fortunately, no spectrometer data, small in size, and also higher in priority, was lost during swingby. 
Table 13 Number of images or spectra per instrument in the mission's final hour and final five minutes

\begin{tabular}{|c|c|c|c|c|c|c|c|c|c|}
\hline & \multicolumn{5}{|l|}{ Final hour ${ }^{\mathrm{c}}$} & \multicolumn{4}{|c|}{ Last five minutes } \\
\hline & $\begin{array}{l}\text { Actual } \\
\text { E2E } \\
\text { test }^{\mathrm{a}}\end{array}$ & Planned $^{\mathrm{b}}$ & Actual & $\begin{array}{l}\text { Actual- } \\
\text { planned }^{b}\end{array}$ & $\Delta(\%)$ & Planned $^{b}$ & Actual & $\begin{array}{l}\text { Actual- } \\
\text { planned }^{b}\end{array}$ & $\Delta(\%)$ \\
\hline VIS & 2,543 & 2,351 & 2,367 & 16 & 0.68 & 147 & 98 & -49 & -33 \\
\hline NIR1 & 1,427 & 1,425 & 1,423 & -2 & -0.14 & 231 & 228 & -3 & -1.3 \\
\hline NIR2 & 1,364 & 1,506 & 1,314 & -192 & -13 & 111 & 105 & -6 & -5.4 \\
\hline MIR1 & 9,868 & 7,351 & 5,256 & $-2,095$ & -29 & 734 & 435 & -299 & -40.7 \\
\hline MIR2 & 9,860 & 7,344 & 2,761 & $-4,583$ & -62 & 735 & 380 & -355 & -48.3 \\
\hline VSP-BM ${ }^{d}$ & 694 & 797 & 627 & -170 & -21 & 61 & 37 & -24 & -39.3 \\
\hline VSP-SM ${ }^{\mathrm{d}}$ & 0 & 0 & 1 & 1 & 0 & 0 & 1 & 1 & N/A \\
\hline NSP1-IF ${ }^{d}$ & 15,921 & 15,846 & 15,924 & 78 & 0.49 & 4,655 & 4,658 & 3 & 0.1 \\
\hline NSP1-HS ${ }^{d}$ & 5,204 & 5,556 & 5,049 & -507 & -9.1 & 400 & 399 & -1 & -0.3 \\
\hline NSP1-DM ${ }^{\mathrm{d}}$ & 12,417 & 12,417 & 12,420 & 3 & 0 & 0 & 0 & 0 & 0.0 \\
\hline NSP2-IF & 11,255 & 11,255 & 11,256 & 1 & 0 & 0 & 0 & 0 & 0.0 \\
\hline NSP2-HS ${ }^{d}$ & 5,313 & 5,666 & 5,157 & -509 & -8.9 & 509 & 508 & -1 & -0.2 \\
\hline NSP2-DM ${ }^{\mathrm{d}}$ & 12,418 & 12,418 & 12,420 & 2 & 0 & 0 & 0 & 0 & 0.0 \\
\hline TLP & $1,426,200$ & $1,445,000$ & $1,300,100$ & $-144,900$ & -10 & 245,830 & 239,126 & $-6,704$ & -2.7 \\
\hline
\end{tabular}

The numbers are the number of images or spectra or TLP samples (divided by 100)

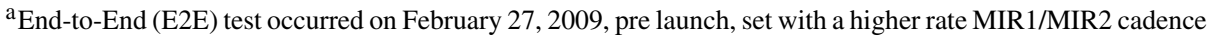

${ }^{b}$ Incorporated the planned lower MIR1/MIR2 rates based on swingby analysis. They were further lowered in the final hour

${ }^{\mathrm{c}}$ These counts also assumed a $59 \mathrm{~m} 09.15 \mathrm{~s}$ sequence run. The actual sequence ran for $54 \mathrm{~m} 18.388 \mathrm{~s}$, or 290.7 s shorter

${ }^{\mathrm{d}}$ Definition of modes: VSP: $\mathrm{BM}=$ Bracket mode, $\mathrm{SM}=$ Single Mode; NSP: $\mathrm{IF}=$ Impact Flash Mode, HS = Hadamard Spectrum Mode, DM = Diagnostic Mode

After realizing the bandwidth stresses and image dropouts were dominated by the larger thermal camera image sizes, the thermal camera sampling rates were changed in the instrument command sequences for the final hour and simulated several times with the payload simulator before being loaded to the spacecraft a few days before end of mission. In the mission's final hour, the compression problem turned out to be about $20 \%$ worse than during the lunar swingby. The payload operations team had anticipated this outcome and had prepared in advance commands to slow both MIR thermal camera rates in real time to keep the margins sufficient to prevent loss of data. Both thermal cameras had their rates slowed down during the final descent. A comparison of the actual data retrieved during the final hour and specifically the last five minutes compared to the planned lower rate sequences is summarized in Table 13. As shown here the majority of the actual data losses, compared to the data plan, were in the MIR cameras. The visible camera was similarly affected, not by deliberate data rate changes, but due to limits in the DHU software (see below). The highest priority instruments, the near infrared spectrometers, were not affected by these sequence changes, experiencing only a $0.1-0.3 \%$ loss of data when compared to the data plan. The larger data loss (39\% by number of spectra) by the UV-visible spectrometer in the final five minutes is described below, a side-effect of the larger bandwidth stress situation affecting the DHU operation. 
Fig. 24 High scene complexity caused compressed images to exceed the maximum telemetry packet size, resulting in image artifacts. The impact plume is distinguishable from the artifacts due to differing noise characteristics. This image is a raw VIS image taken 9 seconds after the Centaur impact

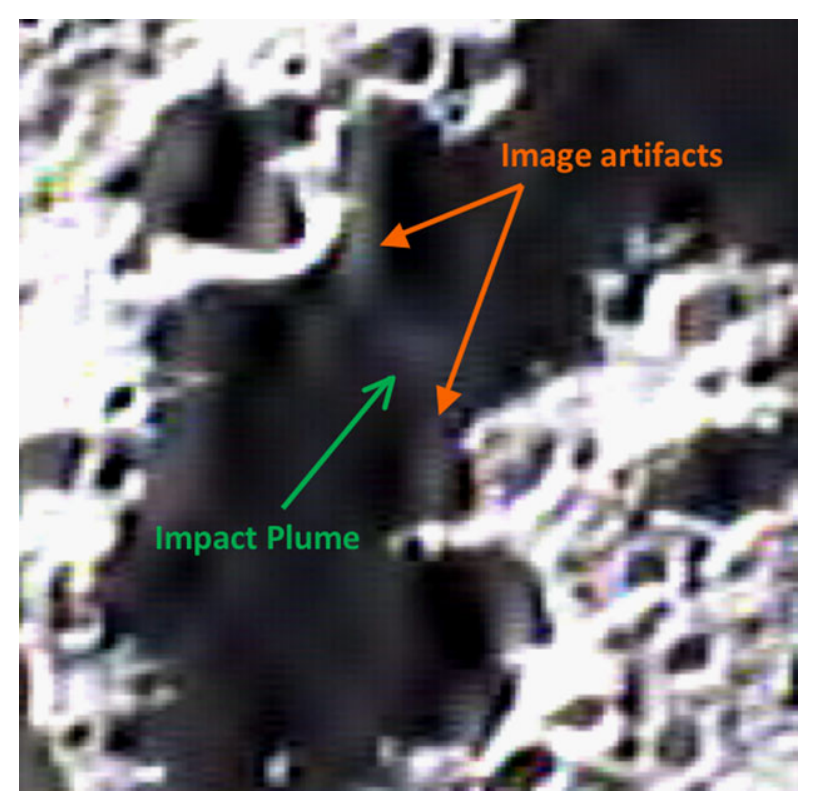

Despite these attempts to keep the bandwidth margins healthy, the DHU remained in a stressed state packaging the camera data. One side effect was that some of the visible camera images were still too large to fit within a single data packet for transmission to Earth. An example of the kind of damaged image that resulted is shown in Fig. 24. This damage cannot be fully corrected because information was lost, but the impact plume can be distinguished from the artifacts because the noise in that region is different.

The root cause in the damaged visible images was found to lie in Ecliptic heritage image compression software. This code had been written to clip the images in compressed form to ensure they always fit within a single data packet or a maximum size of 65536 bytes. The LCROSS payload software design used a wavelet-based compression algorithm, and compressed images that had been clipped removed some information needed to recreate the image accurately. The alternative would have been to split the images across multiple packets and reassemble them on the ground. This certainly could have been done in principle, but doing so would have introduced significant changes to flight-proven heritage software.

In the final hour of the mission, the commanding side of the automatic sequence ran almost perfectly. Only one problem was noted, a symptom of the bandwidth stresses indicated above by the large images. Because the DHU was at its maximum data throughput capacity during the first seconds after impact, one command to change exposure time on the UV-visible spectrometer was delayed and sent during a period when the instrument was not listening. As a result, that command was ignored. This resulted in capturing fewer spectra, but each spectrum now had a longer-than-planned exposure time. Ironically, the longer exposure times turned out to be useful, since the ejecta curtain was much fainter than the models predicted. The unfortunate side effect was a loss of more frequent sampling due to the longer exposures, and hence a decrease in the total number of spectra. Although fewer spectra were obtained, this did not affect the science measurement. The VSP was able to record several independent spectral measurements of the ejecta curtain over four minutes (Schultz et al. 2010). The DHU time-stamping function and error logs helped identify this 
issue in the data. This was an instrument-DHU interaction that had never been seen in any prior testing.

\section{Summary}

The LCROSS instrument payload suite was designed to provide the mission scientists with multiple complementary views of the ejecta curtain created by the Centaur impact. This suite, comprised of ruggedized commercial-off-the-shelf components, provided a solution that met the tight budget and aggressive schedule of this secondary payload. A tailored space qualification test program was developed that met the NASA Class D requirements at the time. When weaknesses were revealed, the team worked closely with the instrument vendor suppliers to improve the robustness of the payload. The LCROSS payload team used open and frequent reviews by NASA project systems engineering and quality assurance to manage and mitigate payload technical risk throughout the project lifetime, including through operations. All nine science instruments aboard the LCROSS shepherding spacecraft successfully collected data during the final descent to the lunar surface after 112 days in space. They also successfully provided the data to answer the mission's science questions. On-orbit calibration proved to be essential to validate pre-launch test data. Turning on the instruments during the lunar swingby, during the first week of the mission, had been intended as a learning experience, but it also proved critically important, revealing a test oversight in estimating the bandwidth. It provided the best operational practice for the impact observations. The LCROSS Class D mission and payload design provides a current successful example where actively managed risk, low-cost approaches to well-defined goals with clearly stated requirements can bring about large science payoffs.

Acknowledgements We thank Diane Wooden, Jennifer Heldmann, David Landis, David Day and Kimberly Mjaseth for their work on calibrating the payload instruments. This research has made use of the SIMBAD database, operated at CDS, Strasbourg, France. We wish to thank the LCROSS payload engineering team Glen Sasaki, Jim Hanratty, Gil Kojima, Dana Lynch, Frank Buchey, Lynn Hofland, Dave Scimeca, and Jerry Wang, the NASA ARC project support Dan Andrews, Leonard Hee, and Robert Barber, and NGST system engineering for their guidance. The LCROSS project was funded by NASA's Exploration Systems Mission Directorate.

\section{References}

APR-8070.2. Ames Procedural Requirements. Class D spacecraft design and environmental test (Effective Date 17 September 2008). Current version can be found at: http://server-mpo.arc.nasa.gov/

ASTM E-490. 2000 American Society for Testing \& Materials (ASTM) standard extraterrestrial solar spectrum reference E-490-00 [Online] (2000). Available: http://rredc.nrel.gov/solar/spectra/am0/

D. Andrews, LCROSS: a high-return, small satellite mission, in Small Satellite Systems and Services, the $4 S$ Symposium, Funchal, Madeira, 31 May-4 June 2010 (2010)

E.S. Barker, C.A. Prieto, T.L. Farnham, D.B. Goldstein, R.S. Nerem, J.V. Austin, J.Y. Shim, A.B. Storrs, and 32 authors, Results of observational campaigns carried out during the impact of lunar prospector into a permanently shadowed crater near the South Pole of the moon. Bull. Am. Astron. Soc. 31, 1583 (1999). American Astronomical Society, DPS Meeting 31, Abstract 59.03

S.W. Brown, B.C. Johnson, S.F. Biggar, E.F. Zalewski, J. Cooper, P. Hajek, E. Hildum, P. Grant, R.A. Barnes, J.J. Butler, Radiometric validation of NASA Ames Research Center's Sensor Calibration Laboratory. Appl. Opt., 4(30), 6426-6443 (2005)

A. Colaprete, P. Schultz, J. Heldmann, M. Shirley, K. Ennico, B. Hermalyn, D. Wooden, W. Marshall, A. Ricco, R.C. Elphic, D. Goldstein, D. Summy, G.D. Bart, E. Asphaug, D. Korycansky, D. Landis, L. Sollitt, The detection of water within the LCROSS ejecta plume. Science 300, 463-468 (2010) 
J. Cooper, NASA GSFC Code 614.4, Calibrations NIST 30 inch Sphere NASA Ames Research Center (2007). Available: http://cf.gsfc.nasa.gov/docs/Cal/Other/Archi/2007/20070518083800Archi.txt

J. Cooper, NASA GSFC Code 614.4, Calibrations NIST 30 inch Sphere NASA Ames Research Center (2008). Available: http://cf.gsfc.nasa.gov/docs/Cal/Other/Archi/2008/20080314105200Archi.txt

D.R. Day, M.A. Butler, M.C. Smith, A. McAlister, E.R. Deutsch, K. Zafiriou, S.D. Senturia, DiffractiveMEMS implementation of a Hadamard near-infrared spectrometer, in The 13th International Conference on Solid-State Sensors, Actuators and Microsystems, Seoul, Korea, June 5-9, 2005 (2005), pp. 12461249

L.R. Gauthier, L.M. Howser, D.T. Prendergast, M.P. Mattix, Radiometric dynamic scene processing for uncooled IRFPAs, infrared imaging systems: design, analysis, modeling, and testing XVII. Proc. SPIE 6207, 241-250 (2006)

G.R. Gladstone, D.M. Hurley, K.D. Retherford, P.D. Feldman, W.R. Pryor, J. Chaufray, M. Versteeg, T.K. Greathouse, A.J. Steffl, H. Throop, J.W. Parker, D.E. Kaufmann, A.F. Egan, M.W. Davis, D.C. Slater, J. Mukherjee, P.F. Miles, A.R. Hendrix, A. Colaprete, S.A. Stern, LRO-LAMP observations of the LCROSS impact plume. Science 300, 472-476 (2010)

E.W. Greisen, M.R. Calabretta, Representations of world coordinates in FITS. Astron. Astrophys. 395, 10611075 (2002)

GSFC-STD-7000. General environmental verification standard GEVS. For GSFC flight programs and projects (2005). Current version can be found at http://standards.gsfc.nasa.gov/gsfc-stds.html

P.O. Hayne, B.T. Greenhagen, M.C. Foote, M.A. Siegler, D.A. Paige, Diviner lunar radiometer observations of the LCROSS impact. Science 300, 477-479 (2010)

J. Heldmann, A. Colaprete, and 67 authors. LCROSS (Lunar Crater Observation and Sensing Satellite) observation campaign: strategies, implementation, and lessons learned. Space Sci. Rev. (2011, this issue). doi:10.1007/s11214-011-9753-4

W. Marshall, M. Shirley, Z. Moratto, A. Colparete, G. Neumann, D. Smith, S. Hensley, S. Hensley, B. Wilson, M. Slade, B. Kennedy, Locating the LCROSS impact crater. Space Sci. Rev. (2011, in press)

MIL-HDBK-343 (USAF). Military Handbook: Design, Construction, and Testing Requirements for One of a Kind Space Equipment. 1 February (1986)

MIL-HDBK-340A (USAF). Military Handbook: Test Requirements for Launch, Upper-Stage, and Space Vehicles (Vol. 1: Baselines). 1 April (1999)

MIL-STD-1540B. Military Standard: Test Requirements for Space Vehicles. 10 October (1982)

I.G. Mitrofanov, A.B. Sanin, W.V. Boynton, G. Chin, J.B. Garvin, D. Golovin, L.G. Evans, K. Harshman, A.S. Kozyrev, M.L. Litvak, A. Malakhov, E. Mazarico, T. McClanahan, G. Milikh, M. Mokrousov, G. Nandikotkur, G.A. Neumann, I. Nuzhdin, R. Sagdeev, V. Shevchenko, V. Shvetsov, D.E. Smith, R. Starr, V.I. Tretyakov, J. Trombka, D. Usikov, A. Varenikov, A. Vostrukhin, M.T. Zuber, Hydrogen mapping of the lunar south pole using the LRO neutron detector experiment LEND. Science 300, 483485 (2010)

Z. Moratto, W. Marshall, M. Shirley, M. Broxton, A. Colaprete, LCROSS derived map products, in NASA Lunar Science Forum, Moffett Field, CA, July 2010 (2010)

NPR 8705.4. NASA procedural requirements risk classification for NASA payloads (revalidated July 9 , 2008). Current version can be found at: http://nodis3.gsfc.nasa.gov/

D.A. Paige, M.C. Foote, B.T. Greenhagen, J.T. Schofield, S. Calcutt, A.R. Vasavada, D.J. Preston, F.W. Taylor, C.C. Allen, K.J. Snook, B.M. Jakosky, B.C. Murray, L.A. Soderblom, B. Jau, S. Loring, J. Bulharowski, N.E. Bowles, I.R. Thoma, M.T. Sullivan, C. Avis, E.M. De Jong, W. Hartford, D.J. McCleese, The lunar reconnaissance orbiter diviner lunar radiometer experiment. Space Sci. Rev. 150, 125-160 (2010a)

D.A. Paige, M.A. Siegler, J. Zhang, P.O. Hayne, E.J. Foote, K.A. Bennett, A.R. Vasavada, B.T. Greenhagen, J.T. Schofield, D.J. McCleese, M.C. Foote, E. DeJong, B.G. Bills, W. Hartford, B.C. Murray, C.C. Allen, K. Snook, L.A. Soderblom, S. Calcutt, F.W. Taylor, N.E. Bowles, J.L. Bandfield, R. Elphic, R. Ghent, T.D. Glotch, M.B. Wyatt, P.G. Lucey, Diviner lunar radiometer observations of cold traps in the Moon's south polar region. Science 300, 479-482 (2010b)

Y. Ralchenko, A.E. Kramida, J. Reader, NISTASD Team, NIST Atomic Spectra Database (version 3.1.5) [Online]. National Institute of Standards and Technology, Gaithersburg, MD (2008). Available: http://physics.nist.gov/asd3 [2010, June 27]

R. Ridenoure, RocketCam systems for providing situational awareness on rockets, spacecraft, and other remote platforms. Proc. SPIE 5418, 94-103 (2004)

M. Saccoccio, R. Wiens, B. Barraclough, J.D. Bernardin, A. Cros, S. Bender, S. Clegg, L. Pares, K. Gasc, D. Kouach, B. Dubois, M. Bouye, J. Thocaven, H. Seran, Y. Parot, R. Orttner, B. Faure, Y. Michel, P. Cais, M. Berthe, R. Perez, R. Stiglich, D. Landis, T. Hale, C. Hayes, C. Lindensmith, T. Elliot, The CHEMCAM instrument on Mars science laboratory: first laser induced breakdown spectroscopy instrument in space, in International Conference on Environmental Systems, Savannah, GA, USA (2009). États-Unis d'Amérique 
T.L. Segura, A.S. Lo, H. Eller, D. Dailey, E. Drucker, J. Wehner, Secondary payloads using the LCROSS architecture, in 41st Lunar and Planetary Science Conference, The Woodlands, Texas, March 1-5, 2010 (2010), p. 2673. LPI Contribution No. 1533

P.H. Schultz, B. Hermalyn, A. Colaprete, K. Ennico, M. Shirley, W. Marshall, The LCROSS cratering experiment. Science 300, 468-472 (2010)

M.F. Skrutskie, R.M. Cutri, R. Stiening, M.D. Weinberg, S. Schneider, J.M. Carpenter, C. Beichman, R. Capps, T. Chester, J. Elias, J. Huchra, J. Liebert, C. Lonsdale, D.G. Monet, S. Price, P. Seitzer, T. Jarrett, J.D. Kirkpatrick, J.E. Gizis, E. Howard, T. Evans, J. Fowler, L. Fullmer, R. Hurt, R. Light, E.L. Kopan, K.A. Marsh, H.L. McCallon, R. Tam, S. Van Dyk, S. Wheelock, The two micron all sky survey (2MASS). Astron. J., 131, 1163-1183 (2006)

H.E. Spence, The Crater Team, Lunar cosmic ray albedo measurements using the cosmic ray telescope for the effects of radiation on the lunar reconnaissance orbiter, in 41 st Lunar and Planetary Science Conference, The Woodlands, March 1-5, 2010 (2010). Abstract \#2659

P.D. Tompkins, R. Hunt, M. D’Ortenzio, J. Strong, K. Galal, J.L. Bresina, D. Foreman, R. Barber, M. Shirley, J. Munger, E. Drucker, Flight operations for the LCROSS lunar impactor mission, in AIAA Space Ops 2010 Conference, Delivering on the Dream (2010) (AIAA, Washington, 2010). Paper 2010-1986

TR-2004 (8583)-1 REV. A. Air force space command: test requirements for launch, upper-stage, and space vehicles. 6 September (2006)

R. Vondrak, J. Keller, G. Chin, J. Garvin, Lunar reconnaissance orbiter (LRO): observations for lunar exploration and science. Space Sci. Rev. 150, 7-22 (2010)

D. Wooden, K. Ennico, A. Colaprete, J. Heldmann, M. Shirley, E. Hildum, P. Grant, LCROSS near-IR spectrometers: characterizing the spectrometers that look for water ice in impact plume radiance, wavelength \& instrument profile calibration using the Ames airborne Sensor Cal Lab (SCL), in NLSI Lunar Science Forum Workshop, Washington, 21-23 July 2009 (2009) 